\title{
The Predictive Value of Platelet Function Point-of-Care Tests for Postoperative Blood Loss and Transfusion in Routine Cardiac Surgery: A Systematic Review
}

\author{
Mate Petricevic ${ }^{1}$ Tomislav Kopjar ${ }^{1}$ Bojan Biocina ${ }^{1}$ Davor Milicic ${ }^{2}$ Kresimir Kolic ${ }^{3}$ Marko Boban ${ }^{4}$ \\ Bosko Skoric ${ }^{2}$ Ante Lekic ${ }^{1}$ Hrvoje Gasparovic $^{1}$ \\ ${ }^{1}$ Department of Cardiac Surgery, University of Zagreb School of \\ Medicine-University Hospital Center Zagreb, Zagreb, Croatia \\ 2 Department of Cardiovascular Diseases, University of Zagreb School \\ of Medicine-University Hospital Center Zagreb, Zagreb, Croatia \\ ${ }^{3}$ Department of Medical Diagnostics, University of Split School of \\ Medicine-University Hospital Center Split, Split, Croatia \\ ${ }^{4}$ Department of Cardiology, University Hospital "Thalassotherapia \\ Opatija," Medical School University of Rijeka and Osijek, Opatija, \\ Croatia

\begin{abstract}
Address for correspondence Mate Petricevic, MD, PhD, Department of Cardiac Surgery, University of Zagreb School of Medicine, University Hospital Center Zagreb, Kispaticeva 12, 10000 Zagreb, Croatia (e-mail: petricevic.mate@gmail.com).
\end{abstract} \\ Thorac Cardiovasc Surg 2015;63:2-20.
}

\begin{abstract}
Keywords

- blood transfusion

- blood

- coagulation/ anticoagulation

- coronary artery bypass grafts surgery

Excessive bleeding after cardiopulmonary bypass ( $\mathrm{CPB}$ ) operations remains to be a persistent problem and weak platelet function certainly contributes to bleeding diathesis. Antiplatelet therapy (APT) is an integral component of perioperative management in patients undergoing cardiac surgery procedures, both with and without use of CPB. In addition to individual variability in platelet function, different preoperative APT administration/discontinuation management further affects platelet function, which in turn may reflect bleeding tendency. However, the impact of drug-induced platelet inhibition on early postoperative bleeding extent remains difficult to predict. Herein, we reviewed the available evidence on the association between platelet function testing values and the extent of bleeding and transfusion requirements in early perioperative period. Currently, the association between platelet function measured by ex vivo assay and the occurrence of bleeding events remains uncertain. The intent of this review is to provide comprehensive literature insight into published evidence, investigating the possibility of platelet function tests to predict bleeding extent as well as transfusion requirements in cardiac surgery patients.
\end{abstract}

\section{Introduction}

Bleeding extent as well as transfusion of allogeneic blood products certainly affects outcome following cardiac surgery procedures. Up to $10 \%$ of patients undergoing cardiac surgery experience excessive postoperative hemorrhage. ${ }^{1}$ Christensen et al investigated the relationship between postoperative

received

October 15, 2013

accepted after revision

March 14, 2014

published online

July 1, 2014

hemorrhage and clinical outcome following cardiac surgical procedures. ${ }^{2}$ Patients who experienced excessive postoperative bleeding had higher 30-day mortality and other adverse outcomes such as stroke, reexploration, intensive care unit stay, and mechanical ventilation. ${ }^{2}$ Those findings are in line with results reported by Dixon et al. $^{3}$ Authors undertook a multivariate logistic regression analysis of the risk factors

(c) 2015 Georg Thieme Verlag KG Stuttgart · New York
DOI http://dx.doi.org/ 10.1055/s-0034-1378191. ISSN 0171-6425. 
associated with mortality in 2,599 consecutive patients undergoing cardiac surgery. ${ }^{3}$ With aim to consider the possibility that chest tube drainage (CTD) may in itself be harmful, the risk factors examined included the volume of CTD at 24 hours. $^{3}$ CTD was the strongest independent predictor of mortality $(p<0.001) .^{3}$ Although results reported by Dixon et $\mathrm{al}^{3}$ have numerous implications for surgical practice, the question how to predict and prevent excessive CTD remains challenging. The meticulous hemostatic surgical technique is mandatory ${ }^{4}$; however, sometimes it is insufficient to achieve adequate hemostasis, probably due to coagulopathic component of bleeding.

Platelet function plays important role in pathogenesis of hemostatic disorder and consequent bleeding diathesis in cardiac surgery patients. Platelet function may be considered as a continuous variable that expresses widespread range among individuals. Widespread range of platelet activity among individuals arises from different factors such as (1) widespread interindividual variability in inherent platelet activity, (2) widespread variability in platelet inhibitory response to antiplatelet therapy (APT), and (3) individual ability to recover platelet function after APT cessation, which is also influenced by timing of discontinuation and type of drug used. There is evidence that heritable factors play a major role in determining platelet aggregation ${ }^{5}$ and such a variability in platelet aggregation may in some degree explain proclivity toward bleeding as well as ischemic events. In addition to heritable factors, platelet function is dominantly influenced by APT. Patients experiencing the therapeutic effects of APT and requiring cardiac surgery are at risk for adverse bleeding events and transfusion requirements. Patients receiving APT have different degrees of platelet inhibition. Widespread variability in platelet responses to the most commonly prescribed antiplatelet drugs such as acetylsalicylic acid (ASA) and clopidogrel (CLO) have been established by various platelet function assays. ${ }^{6}$ The bleeding risk has recently become accentuated by the widely prevalent dual APT with ASA and CLO. Despite the current guidelines, many centers continue with APT till the day of surgery, disregarding the recommendations for a drug-free interval before surgery. For example, data from 2,858 acute coronary syndrome patients in the CRUSADE (Can Rapid risk stratification of Unstable angina patients Suppress ADverse Outcomes with Early implementation of the ACC/AHA Guidelines) initiative demonstrated that $87 \%$ of CLO-treated patients underwent coronary artery bypass graft (CABG) surgery $\leq 5$ days after treatment with a consequently increased blood transfusion requirement. ${ }^{7}$ Notably, 5 to $15 \%$ of patients with an acute coronary syndrome require an urgent cardiac operation with recently administered antiplatelet drugs. ${ }^{7}$

The considerable heterogeneity in platelet inhibitory response to APT makes it difficult to safely use arbitrary interval for drug discontinuation before surgery without incurring excessive thrombotic or bleeding risks with premature versus too late discontinuation. Therefore, an individual approach in preoperative assessment of the bleeding risks is warranted. Bedside-suitable platelet function testing could play an important role in the prediction and prevention of excessive bleeding after cardiopulmonary bypass (CPB). Notably, conventional coagulation tests have been shown to be unreliable in predicting postoperative bleeding., ${ }^{8,9}$ Platelet function testing presents a kind of phenotyping approach that provides quantification of platelet function by summarizing the effects of all previously mentioned covariates that influence platelet activity. Theoretically, platelet function tests could guide clinical decision making in patients on APT. Evaluating the efficiency of platelet inhibition in the preoperative settings offers to identify patients in whom excessive bleeding is likely and often preventable. ${ }^{10}$ Velik-Salchner et al ${ }^{10}$ showed that bedside-suitable platelet function test was capable to discriminate patients according to preoperative APT exposure $^{10}$ as well as to detect CPB-induced changes in platelet aggregation. $^{10}$

Hemostatic disorders became more apparent with perioperative use of novel antithrombotic drugs such as prasugrel and ticagrelor. Prasugrel, third-generation thienopyridine, achieves more rapid and greater platelet inhibition than CLO and is substantially associated with higher rates of major and minor bleeding in cardiac surgery patients. ${ }^{11,12}$ Ticagrelor, novel, nonthienopyridine antiplatelet drug achieves more pronounced inhibition of platelet function than CLO. ${ }^{11}$ More potent antiplatelets to which patients are more often exposed in close proximity to surgery require prudent hemostatic management. Despite the fact that novel antithrombotic drugs achieve greater inhibition of platelet aggregation with a lower rate of nonresponders, ${ }^{13}$ it is obvious that antiplatelet drugs act in a wide range of inhibition. Such variability may occur during the initiation of therapy, steadystate administration, as well as after discontinuation of treatment with variable interval needed to achieve recovery after drug discontinuation. Consequently, platelet function testing seems reasonable. Use of platelet function testing may help improve hemostatic management by stratification of patients according to different levels of platelet inhibition, thus different risks of excessive bleeding due to platelet dysfunction. In this way, it would be possible to determine "safe window" of platelet inhibition that would permit safe surgery in regard to bleeding risk. In certain proportion of patients, APT could be discontinued even before the recommended waiting period, and in this way it could be possible to minimize both bleeding and ischemic events occurrence. Cangrelor, a reversible intravenous P2Y12 inhibitor, has a fast onset of action and very short half-life ${ }^{14}$ which allows for precise temporal programming of platelet inhibition and has recently been used for bridging APT in patients scheduled for cardiac surgery. ${ }^{15}$ Short-acting (eptifibatide and tirofiban) and long-acting (abciximab) GPIIB/IIIA inhibitors cause profound platelet inhibition. As some patients on GPIIB/IIIA inhibitors require emergent surgery, particular attention should be paid to patients on long-acting agents such as abciximab. New anticoagulation agents such as factor Xa inhibitor rivaroxaban and apixaban and direct thrombin inhibitor such as dabigatran may further amplify hemostatic disorder in cardiac surgery patients. There is some evidence that point-of-care devices are not suitable for assessment of hemostatic disturbances caused with factor Xa inhibitor 
administration. ${ }^{16}$ However, further elaboration on this issue goes far beyond the scope of this review article.

In this article, we reviewed available evidence on the utility of platelet function testing in assessment of excessive bleeding as well as transfusion requirements in patients undergoing cardiac surgery. The intent of this review is to provide comprehensive insight into published evidence investigating whether platelet function tests are capable to predict excessive bleeding and transfusion requirements in cardiac surgery patients by matching quantified platelet function with evaluated endpoints such as bleeding extent and transfusion requirements.

\section{Methods}

PubMed and Medline were searched using predefined search terms such as "platelet function testing," "bleeding cardiac surgery," and "transfusion cardiac surgery" using Boolean logic operators [AND] and [OR]. Abstracts were evaluated, and relevant full-text articles were obtained. In addition, reference lists of selected publications were analyzed for additional linking studies. Articles that addressed the relation between platelet function testing and bleeding outcomes as well as transfusion requirements in cardiac surgery patients were included in review. During literature review, we aimed to plot platelet function testing against different endpoints. Excessive bleeding was defined as proposed by authors in respective manuscripts. Different authors proposed different ways to define excessive bleeding. Excessive bleeding as an endpoint was mainly defined in respect to extent of CTD in certain amount of time. Different amounts of CTD revealed in different timeframes were used and patients were defined as excessive bleeders if their CTD exceeded predefined cutoff value for excessive bleeding. The other important endpoint was "transfusion requirements." Through searched literature, we observed transfusion requirements for different procoagulant blood components transfusion. Although transfusion requirements for platelet concentrate would have clear rationale, we decided to assess for all procoagulant blood components transfusion requirements. In noninterventional studies where attending anesthesiologists and surgeons are unaware of platelet function testing results and where decision to transfuse procoagulant blood components relies either on institutional algorithm or solely on the clinical judgment, weak platelet function may cause wet field which in turn may be treated with different types of procoagulant blood components, depending on the institutional algorithm or preference of clinicians blinded to platelet function results.

\section{Monitoring of Platelet Function in Cardiac Surgery Patients}

Therapeutic approach to surgically or coagulopathically induced bleeding is quite different. Bedside assessment of blood hemostatic properties has several advantages over conventional coagulation testing. First of all, turnaround time from sampling blood to obtaining results is often crucial. Although point-of-care tests show more variation and lower precision, the focus should be on the accuracy sufficient to make appropriate clinical decisions timely. Despite the fact that standard coagulation testing is focused more on accuracy than on turnaround time, ${ }^{17}$ conventional coagulation testing failed to predict postoperative bleeding in cardiac surgery patients. $^{8,9}$ In addition, conventional laboratory tests are unable to decompose multifactorial pathogenesis of hemostatic disorder as described by Paparella et al. ${ }^{18}$

Thromboelastography (TEG) has been used in hospital laboratories since its development by Hartert in 1944 at Heidelberg University. Spiess et al published the first study evaluating the relationship between TEG and bleeding extent after cardiac surgery. ${ }^{8}$ In small study cohort ( 38 patients), TEG was predictor of postoperative hemorrhage. ${ }^{8}$ However, platelet function was only indirectly assessed because modified TEG with platelet function mapping was not available. Nowadays, the two most commonly employed point-of-care devices for assessment of viscoelastic blood properties are TEG (Haemoscope Corporation, Niles, Illinois, United States) and the ROTEM thromboelastometer (TEM International GmbH, Munich, Germany). Differences as well as similarities between TEG and thromboelastometry systems should be briefly elaborated. Both systems measure viscoelastic properties of blood clot by employing a vertical pin held in the blood sample, contained within a cup. ${ }^{19}$ Technical aspects of differences between two systems as well as advantages and disadvantages of one system over the other are published by Jackson et al in "head to head" comparison. ${ }^{19}$ Although these two devices share a very similar concept, divergent results on similar samples were reported by Nielsen. ${ }^{20}$ of more importance, in particular in this review article, should be noted that only TEG provides platelet function mapping by adding specific platelet function agonists that allow for quantification of platelet function through different pathways. In addition to TEG platelet mapping, various bedside-suitable, pointof-care platelet function tests have been increasingly used. Nowadays, several devices are available. Detailed description of mostly used current platelet function testing devices has been already published. ${ }^{21}$

\section{Association between Platelet Function Tests Findings and Bleeding Outcomes and Transfusion Requirements}

Conflicting data have been published regarding ability of point-of-care platelet function assays to predict bleeding extent as well as transfusion requirement after cardiac surgery.

Poston et al investigated the predictive value of platelet function testing in assessment of bleeding and thrombotic events after off-pump CABG surgery. ${ }^{22}$ Two point-of-care platelet function devices were used: (1) TEG assay using arachidonic acid agonist (TEG platelet mapping [Haemoscope Corporation, Niles, Illinois, United States]) and (2) whole blood aggregometry (WBA) (model592A; Chronolog, Havertown, Pennsylvania, United States). Intraoperative amount of bleeding significantly correlated with the decrease in platelet function as assessed by WBA $(r=0.42, p<0.05)$, but not TEG 
( $p=\mathrm{ns}$ ). The same TEG device failed to significantly correlate with 24-hour CTD in study by Carrol et al. ${ }^{23}$ It should be noted that patients on APT were excluded from the study a priorily, ${ }^{23}$ thus making the cohort to be of low bleeding risk in terms of platelet dysfunction. The role of platelet function testing in group of patients not exposed to APT seems to be questionable. The fact is that platelet function testing may be of the greatest benefits in patients who are exposed to APT. On the contrary, Preisman et al reported TEG parameter using adenosine diphosphate (ADP) agonist to significantly predict bleeding extent ${ }^{24}$ with a sensitivity of $78 \%$ and specificity of $84 \%$. Comparison between patients with normal and abnormal TEG ADP test revealed larger amount of 24-hour CTD in group of patients with abnormal TEG ADP test. ${ }^{24}$ In addition, the same TEG parameter significantly correlated with transfusion of platelet concentrate (Spearman rho $=-0.75$, $p=0.02$ ). Kwak et al introduced an idea about timing of surgery according to TEG platelet mapping results for patients with CLO administered in proximity to surgery. ${ }^{25}$ Patients were divided into three groups according to tertiles of distribution of the percentage of platelet inhibitory response to CLO therapy. ${ }^{25}$ Patients in the third tertile (the most pronounced platelet inhibition) had a significantly greater amount of postoperative CTD. ${ }^{25}$ Significantly more patients in the third tertile required packed red blood cells and freshfrozen plasma transfusion with higher number of units transfused than those patients with higher residual platelet reactivity following APT administration. ${ }^{25}$ Receiver operating characteristic (ROC) analysis revealed optimal cutoff value for postoperative transfusion requirement as $70 \%$ platelet inhibitory response to $\mathrm{CLO}^{25}$ Accentuated platelet inhibitory response to CLO, but not discontinuation date and preoperative drug-free interval, remained independent risk factor for transfusion requirements. ${ }^{25}$ Weitzel et al conducted prospective observational study using TEG platelet mapping with ADP, arachidonic acid, and collagen as agonists. ${ }^{26}$ TEG platelet mapping assay with collagen as activator (MAcollagen) was found to be the only parameter significantly lower in high bleeding groups in both pre- and post-CPB setting. Furthermore, pre- and post-CPB MAcollagen significantly correlated with 24-hour CTD extent, while only pre-CPB MAaa (maximum amplitude; arachidonic acid used as platelet agonist) (Pearson correlation coefficient $(r),-0.324 ; p=0.041$ ) correlated with 24-hour CTD. ${ }^{26}$ Although MAadp (maximum amplitude; adenosine di-phosphate used as platelet agonist) did not correlate significantly, it is worthwhile to mention that recent preoperative CLO exposure was exclusion criterion which in turn may raise the question whether the obtained results would be different in lower range of MAadp values expected in patients exposed to CLO preoperatively. Mahla et al conducted the first prospective interventional study of a platelet function measurement-based strategy to reduce bleeding and waiting time in CLO-treated patients undergoing CABG (TARGET-CABG study). ${ }^{27}$ Platelet function testing was performed using TEG with ADP agonist. ${ }^{27}$ Patients receiving CLO were divided into three groups according to the platelet function test results and the surgery timing was adjusted for each group according to platelet reactivity as follows: MA-ADP $>50 \mathrm{~mm}$-surgery within 1 day; MA-ADP, 35 to $50 \mathrm{~mm}$-surgery within 3-5 days; and MA-ADP $<35$ $\mathrm{mm}$-surgery after 5 days following CLO discontinuation. All three subgroups along with CLO-naive patients were comparable for the primary (CTD at 24 hours) and secondary endpoints (total number of transfused red blood cells). ${ }^{27}$ Such a strategy allowed for approximately $50 \%$ reduction in waiting time than recommended in the current guidelines, ${ }^{28,29}$ suggesting the fact that uniform waiting period for discontinuation of antiplatelet drugs ${ }^{28,29}$ as suggested in guidelines may be actually obsolete.

With specific aim to evaluate whether Hemostatus-platelet-activating factor clotting time (PACT; Hemostatus, Medtronic, Inc., Parker, Colorado, United States)-device findings correlate with postoperative blood loss, Despotis et $\mathrm{al}^{30}$ conducted prospective observational study. ${ }^{30}$ In a cohort group of 150 patients undergoing cardiac surgery requiring CPB, PACT significantly $(r=-0.85)$ correlated with 4 hours CTD. ${ }^{30}$ Authors concluded that PACT may be a useful tool for the prediction of excessive bleeding. ${ }^{30}$ Although using the same device in similar research and clinical setting, the study of Ereth et al $^{31}$ showed substantially opposite results to those published by Despotis et al. ${ }^{30}$ PACT values obtained after protamine administration correlated weakly with 4 hours CTD $(r=-0.30, p=0.014)$. However, the correlations between postprotamine PACT values and 24-hour CTD as well as with transfusion requirements were not significant. ${ }^{31}$ PACT had comparable results to routine laboratory coagulation tests in the prediction of excessive hemorrhage and authors did not support its use in regular clinical practice. Furthermore, Ereth et $\mathrm{al}^{32}$ conducted another prospective study that duplicated study settings of Despotis et $\mathrm{al}^{30}$ with aim to determine the relation between PACT performed within 1 hour following intensive care unit arrival and blood loss. ${ }^{32}$ Again, PACT values did not correlate with blood loss, ${ }^{32}$ while prothrombin time (PT) strongly correlated with CTD $(p<0.0001){ }^{32}$ Notably, only PT correlated with transfusion of platelets and fresh-frozen plasma. ${ }^{32}$ However, this particular correlation should be interpreted with caution because attending physicians were unaware of only PACT results, but authors did not describe specific hemostatic protocol. Thus, those correlations are expected if procoagulant blood products administration was targeted after PT values.

Dietrich et al conducted prospective study with aim to investigate the relationship between platelet function tests (four different methods: TEG, modification of impedance aggregometry, PFA-100 [Dade Behring, Schwalbach, Germany], and platelet function PAF test) and postoperative bleeding extent in cardiac surgery patients. ${ }^{33}$ Postoperative CTD could not be predicted by platelet function tests performed preoperatively. ${ }^{33}$ However, this findings should be interpreted cautiously because the study enrolled a total of 16 consecutive patients, thus probably being underpowered. ${ }^{33}$ Wahba et $\mathrm{al}^{34}$ conducted prospective comparative study enrolling 40 patients scheduled for elective cardiac surgery. PFA-100 and Hepcon HMS (Medtronic, Dusseldorf, Germany) were compared in the assessment of bleeding prediction to each other and with conventional laboratory and operative 
data. ${ }^{34}$ Preoperative PFA-100 closing time significantly correlated to total blood loss $(r=0.41, p=0.022)$. On the contrary, preoperative Hepcon HMS data failed to correlate with postoperative blood loss $(r=0.18, p=0.37) .{ }^{34}$ Although PFA-100 was found to significantly correlate to CTD, authors considered study to be negative because the correlation of blood loss with PFA-100 was not greater than with the results of conventional and simple coagulation tests such as platelet count, D-dimers, or duration of $\mathrm{CPB} .{ }^{34}$ Using PFA-100 device, Slaughter et al conducted prospective study on 58 adults undergoing primary elective CABG. ${ }^{35}$ Collagen/ADP closure time, measured both preoperatively and 15 minutes after protamine administration failed to correlate with either 6 or 12 hours CTD. Collagen/ADP closure time at 15 minutes after protamine administration did not significantly differ between patients transfused or not transfused with platelet concentrate. ${ }^{35}$ In detecting "bleeders," PFA-100 was found to have positive and negative predictive values of 18 and $98 \%$, respectively. ${ }^{35}$ Those results direct authors to conclude that PFA-100 may be useful in identifying patients unlikely to benefit from platelet transfusion, therefore help to avoid unnecessary platelet transfusion. ${ }^{35}$ Forestier et al compared PFA-100 and Hemostatus point-of-care platelet function tests in study cohort divided into two groups with respect to excessive bleeding presence following arrival to intensive care unit. ${ }^{36}$ Patients were not exposed to APT at least for 7 days before surgery, and additionally were excluded from study if diffuse bleeding occurred without identified surgical source of bleeding after protamine administration. ${ }^{36}$ Platelet function tests were performed following arrival to ICU, either when excessive hemorrhage was diagnosed or after 3 hours following arrival to ICU. ${ }^{36}$ There were no significant differences in Hemostatus and PFA-100 parameter values between "bleeders" and "nonbleeders." ${ }^{36}$ In addition, both Hemostatus and PFA-100 parameter values did not correlate with amount of CTD. ${ }^{36}$ Based on observed overall lack of correlation between platelet function tests and CTD, authors suggested that these platelet function assays should not be routinely used in cardiac surgical patients. ${ }^{36}$ However, they presumed that these tests would be more efficient for patients considered to be at higher risk of bleeding. ${ }^{36}$ Fattorutto et al conducted prospective study with aim to evaluate if PFA100 predicts postoperative blood loss. ${ }^{37}$ PFA-100 failed to predict postoperative bleeding. ${ }^{37}$ Several issues have to be discussed in this article by Fattorutto et al. ${ }^{37}$ Study recruited 70 patients with normal coagulation function and platelet count in whom APT had been withdrawn for at least 5 days before surgery. It is obvious that study was performed in nonbleeding patients group because mean total CTD was $495 \pm 301 \mathrm{~mL}$ and the incidence of excessive CTD was found to be only $6 \%$. Therefore, one may expect that almost every test must fail to predict excessive bleeding in patients who are not bleeding excessively. Cammerer et al conducted prospective study enrolling 255 patients with aim to evaluate weather modified rotational thromboelastometry (ROTEM) (ROTEG [TEM International GmbH, Munich, Germany]) and platelet function analyzer PFA-100 were predictive of postoperative blood loss. ${ }^{38}$ ROTEG and PFA-100 tests performed after CPB were significantly different between patient groups divided with the respect to the presence of excessive postoperative blood loss. ${ }^{38}$ In contrast, preoperative ROTEG findings failed to predict postoperative blood loss. ${ }^{38}$ Noteworthy, abnormal bleeding was defined in two ways, ${ }^{38}$ and the definition of excessive bleeding influenced the ability of PFA-100 to distinguish patients according to bleeding diathesis. ${ }^{38}$ While preoperative PFA-100 showed significant differences between patient groups divided according to the extent of $750 \mathrm{~mL}$ of 6-hour postoperative bleeding, the differences were not present if patients were divided according to study protocol data (upper quartile of blood loss in respective cohort group). ${ }^{38}$ Finally, reported high negative predictive values suggest the role of point-of-care tests in bleeding prediction and decision-making process by identifying patients who will tend to not bleed caused by hemostatic disorder, which direct management of possible bleeding to surgical treatment. ${ }^{38}$

Gerrah et al investigated the possibility of bleeding prediction using cone and plate(let) analyzer (CPA; Impact-R, DiaMed, Cressier/Morat, Switzerland) in cardiac surgery patients. $^{39}$ The major advantage of CPA over the rest of platelet function analyzers is the fact that CPA measures platelet function as well as the interaction between platelets and von Willebrand factor. Patients with pathologic values of CPA parameters had both the higher incidence of excessive postoperative bleeding (>965 mL, 44 vs. 0\%) and higher amount of blood loss $(908 \pm 322 \mathrm{~mL}$ vs. $337 \pm 78 \mathrm{~mL})$ indicating preoperative platelet function as assessed with $\mathrm{CPA}$ as an independent risk factor determining adverse bleeding events. $^{39}$

Solomon et $\mathrm{al}^{40}$ used two point-of-care platelet function analyzers (multiple electrode aggregometry [MEA], Multiplate, Verum Diagnostica GmbH and Dynabyte Informationssysteme $\mathrm{GmbH}$, Munich, Germany, and CPA-Impact $\mathrm{R}$ (DiaMed, Cressier/Morat, Switzerland) with aim to investigate which test is superior in preoperative assessment of platelet concentrate transfusion. MEA was found to be more useful in predicting patients prone to intraoperative platelet concentrate transfusion. ${ }^{40}$ Rahe-Meyer et al provided valuable contribution to evaluation of relationship between MEA results and bleeding extent as well as platelet concentrates transfusion. ${ }^{41}$ MEA findings were divided into tertiles of value distribution. ${ }^{41}$ There were no significant differences between the low and the high tertile of MEA tests with respect to 24-hour drainage volume. ${ }^{41}$ Preoperative MEA ADP and collagen tests were predictive of platelet concentrate transfusion. ${ }^{41}$ Authors concluded that platelet function assay offers the possibility of an early estimation of the risk of transfusion. ${ }^{41}$ In our opinion, this contribution was very valuable because prediction of platelet concentrate transfusion allows for timely hemostatic intervention. In this study, attending clinicians were blinded for platelet function tests results; however, platelet concentrate administration was a first-line therapy in patients considered for hemostatic interventions with known recent exposure to APT. However, the question whether the application of MEA may reduce the amount of platelet concentrate transfusion by targeted administration 
remained uncertain. ${ }^{41}$ Reece et $\mathrm{al}^{42}$ conducted study with aim to determine whether platelet function disorder as assessed by MEA would predict excessive bleeding and blood transfusion. The study conducted in prospective, clinicianblinded fashion enrolled 44 patients. Although CTD was measured hourly for 12 hours and until chest tube removal, authors did not report correlations between CTD extent and platelet function test values. ${ }^{42}$ MEA showed significant differences between transfused and nontransfused patients during chest closure, ADP test ( 18 vs. $29 \mathrm{U} ; p=0.01$ ), and TRAP test (65 vs. $88 \mathrm{U} ; p=0.01$ ), respectively. ${ }^{42}$ Moreover, authors performed ROC $^{43}$ analysis with aim to detect the cutoff value of MEA tests that suggest transfusion requirements. ${ }^{42}$ Measured ROC areas under the curve (AUC) were small ( 0.691 for TRAP test and 0.674 for ADP test) which consequently results in weak prediction models of ROC analysis. ${ }^{42}$ Threshold effect for transfusion outcome was $100 \mathrm{U}$ (TRAP test) and $31 \mathrm{U}$ (ADP test) with specificity values 1 for both tests, but low sensitivity values of 0.333 and 0.267 , respectively. ${ }^{42}$ However, high specificity for observed cutoff values allows for determination of patients who will probably be free of transfusion. ${ }^{42}$ Another study investigating association between MEA and postoperative bleeding and platelet transfusion was conducted by Ranucci et al. ${ }^{44}$ A total number of 87 patients with preoperatively administered thienopyridines were retrospectively analyzed. ${ }^{44}$ ROC analysis was performed and ADP test value of 31 AUC was predictive of excessive bleeding with a sensitivity of $72 \%$ and a specificity of $66 \%{ }^{44}$ High negative predictive value of $92 \%$ suggested the possible role of platelet function test in decision-making algorithm by directing hemostatic management toward surgical hemostasis in cases of normal platelet function test values. Authors described positive relationship between ADP test and platelet transfusion; however, this should be interpreted cautiously because the study was retrospective and ADP test value less than $40 \mathrm{AUC}^{44}$ influenced hemostatic management in respective study cohort. Petricevic et al investigated the possibility of excessive bleeding prediction using MEA in 211 patients following CABG. ${ }^{45}$ MEA was performed preoperatively using ASA-sensitive (ASPI test) and thienopyridines-sensitive (ADP test) platelet function tests. ${ }^{45}$ Both ASPI $(p=0.014)$ and ADP $(p=0.003)$ tests correlated significantly with 24-hour $\mathrm{CTD}^{45}$ and ROC analysis found ASPI test value of $<20$ AUC, as well as ADP test value of $<73$ AUC to be bleeder determinants. ${ }^{45}$ The same research group conducted another prospective study recruiting 148 patients who required elective cardiac surgery. ${ }^{46}$ In this study, use of MEA was supplemented with ROTEM and tests were performed simultaneously in three perioperative time points. The important finding of this study was that the strongest correlations between MEA and ROTEM were observed at third time point of blood sampling (after protamine administration). ${ }^{46}$ Thus, not only preexisting platelet function (influenced by inherent activity and/or APT) but also platelet function altered by CPB and surgical trauma itself may more precisely reflect bleeding tendency. ${ }^{46}$ Weber et al conducted, to our best knowledge, the only one prospective, randomized clinical trial evaluating the efficacy of point-of-care devices in coagulopathic cardiac surgery patients. ${ }^{47}$ Two hemostatic transfusion managements were compared: control group was managed using transfusion algorithm based on conventional laboratory findings, while transfusion management in interventional group was directed according to findings of two point-of-care devices (TEM and MEA). ${ }^{47}$ Study was terminated earlier because the planned interim analysis of the primary outcome (the number of units of packed red blood cells administered in the first postoperative 24 hours) revealed significant group differences favoring interventional group. ${ }^{47}$ In addition, patients in the conventional group lost more blood in early postoperative phase. ${ }^{47}$ We may conclude that point-of-care directed transfusion management resulted with targeted and reduced amount of allogeneic products transfused followed by lower extent of postoperative bleeding. Recently, Schimmer et $\mathrm{al}^{48}$ published retrospective study ${ }^{48}$ with aim to find out whether platelet function tests results reflected postoperative bleeding extent or transfusion requirements. ${ }^{48}$ Put briefly, decreased preoperative MEA platelet function test values were associated with increase in postoperative blood transfusion requirements. ${ }^{48}$ None of the MEA tests performed preoperatively correlated with postoperative bleeding extent. ${ }^{48}$ Di Dedda et al published retrospective analysis of prospectively collected data ${ }^{49}$ with aim to assess the platelet inhibitory response to thienopyridines, to assess the dynamics of platelet function after thienopyridines cessation, and finally to evaluate the relationship between platelet function test values and bleeding and transfusion outcomes. ${ }^{49}$ Using repetitive measurements of MEA ADP test, authors have found that patients with adequate preoperative platelet inhibitory effect revealed with thienopyridines have the mean ADP test increase of $12 \mathrm{U} /$ day following drug discontinuation. ${ }^{49}$ Those data are very valuable because authors described platelet recovery dynamics following thienopyridines discontinuation. ${ }^{49}$ The same research group (SCORE research group) previously defined cutoff value of ADP test 31 AUC to delineate excessive bleeding tendency after cardiac surgical procedures. ${ }^{44}$ Therefore, known cutoff value of 31 AUC, together with expected daily recovery dynamics of 12 AUC per day, allows for tailored preoperative management of thienopyridines discontinuation. On the other hand, if patient on thienopyridines treatment has value of ADP test above cutoff value, it seems reasonable to proceed with surgery without thienopyridines-free interval of 5 days as proposed by guidelines. ${ }^{28}$ Considering bleeding outcomes, the last ADP test value before surgery was significantly associated with postoperative bleeding $(p=0.002) .{ }^{28}$ Finally, authors concluded that platelet function testing immediately before surgery is determinant of postoperative bleeding and requirement for platelet concentrates transfusions. ${ }^{49}$ As the study was retrospective analysis of prospectively collected data, results (especially for platelet concentrate transfusion in thienopyridines-responsive patients subgroup) should be interpreted cautiously because ADP test value was a part of decision-making process, thus creating bias. ${ }^{28}$

Alström et al investigated platelet function in patients undergoing CABG with recent preoperative ASA and CLO exposure (within 3 days $)^{50}$ using flow cytometry including 
VASP phosphorylation and two bedside-suitable point-ofcare analyzers, VerifyNow System (Accumetrics, San Diego, California, United States) and the TEG platelet mapping device. ${ }^{50}$ The authors sought to evaluate whether preoperatively performed assessment of platelet function could predict the risk of bleeding and transfusion requirements following cardiac surgical procedures. ${ }^{50}$ VerifyNowP2Y12 was the only platelet function assay that showed significant correlations with total blood loss (Spearman rho, 0.29; $p=0.03$ ) and the total number of red blood cell transfusions $(r=0.43, p<0.01)$. Notably, none of the above-mentioned assays predicted transfusion of platelet concentrate and fresh-frozen plasma. ${ }^{50}$ Interestingly, TEG platelet mappingMAadp test revealed Spearman rho of only 0.01 in correlation with total blood loss, ${ }^{50}$ which was substantially opposite to findings reported by Preisman et $\mathrm{al}^{24}$ who found the same device to be of clinical usefulness in the prediction of postoperative bleeding. ${ }^{24}$ Very recent study by Rosengart et $\mathrm{al}^{51}$ evaluated the ability of platelet function testing to identify CABG patients at increased risk for bleeding and transfusion outcomes. ${ }^{51}$ Study enrolled patients who underwent isolated CABG with documented preoperative CLO administration. ${ }^{51}$ Preoperative platelet function was assayed within 24 hours of surgery by measuring P2Y12 receptor blockade with the VerifyNow system (Accumetrics). Patients were divided according to the presence of higher platelet reactivity units (PRU) ( $\geq 237$ PRU) or lower platelet reactivity ( $<236$ PRU). Lower PRU subgroup was more likely to bleed excessively (odds ratio [OR], 1.99; 95\% confidence interval [CI], 1.023.88; $p=0.04$; c-index, 0.584) and to be transfused with procoagulant blood components (OR, 2.33; 95\% CI, 1.2-4.54; $p=0.01$; c-index, 0.603). In addition, authors performed exploratory analysis in which patients were divided into three groups according to PRU value distribution (high (> 290 PRU), intermediate (200-290 PRU), and low ( $<200$ PRU). Odds of having excessive CTD and procoagulant blood component increased significantly as an incremental function of exploratory patients grouping according to PRU value distribution (OR, 2.26; $p<0.001$ ). Authors concluded that the risks of bleeding and transfusion outcomes can be easily predicted using point-of-care platelet function testing, ${ }^{51}$ although results should be interpreted cautiously due to retrospective study design.

\section{Discussion}

Bleeding in cardiac surgery is multicausal, and several factors such as comorbidities, preoperative medications, surgical technique, and perioperative hemostatic management, as well as the complexity of surgical procedure influence the extent of postoperative blood loss.

Thus, it seems that due to multicausality in pathogenesis of excessive bleeding following cardiac surgery, attempts to create a universal hemostatic agent will certainly fail.

Several platelet function assays are available, and paucity of data exists regarding its clinical utility, but different assays and different study designs hamper the meaningful comparisons. In general, platelet function testing cutoffs used among different centers are derived from manufacturers, on the clinical leaders' opinion, or are based on the retrospective/ prospective studies conducted in respective centers to delineate clinical outcomes (bleeding, transfusion, and adverse clinical outcomes). However, there is shortage of generally applicable cutoffs that could be reproduced and implemented by worldwide centers. This shortage arises from the lack of prospective multicentric trials that would first define the cutoffs for bleeding events, transfusion requirements, as well as for ischemic events.

Put briefly, to be clinically useful, a platelet function assay must fulfill several criteria: (1) The platelet function test should be validated in large number of specific patient groups. Stratification of patients according to the respective risks should be feasible, and, if possible, platelet function assays should provide "safety window" range of values that should be targeted when deciding on preoperative APT administration and/or discontinuation. (2) The assay should be bedside suitable, user friendly, and should have as short as possible "turnaround time" (time from blood sampling to obtaining results) to provide timely detection of risk for bleeding and subsequent risk of transfusion which would in turn allow for timely hemostatic interventions. Such an approach requires studies to be conducted in prospective fashion to determine whether the platelet function test can identify patients at risk for a particular adverse outcome (excessive bleeding, excessive allogeneic blood products transfusion requirements, adverse ischemic events) using ROC curve $^{43}$ analysis.

When assessing the role of platelet function tests for prediction of bleeding and transfusion outcomes, some methodological considerations should be addressed. Different study designs were used in different investigations with aim to evaluate association between platelet function test results and bleeding extent and transfusion requirements.

Prospective observational studies are capable to provide the most accurate cutoffs with sensitivity and specificity that are best possible in clinical settings. Experimental study setting is not ethically acceptable. For example, although prospective studies using platelet function tests should be "clinician blinded," patients receive hemostatic therapy according to regular clinical protocols and procoagulant blood components administration certainly attenuate correlations coefficient between measured platelet function test values and observed bleeding extent. Second, cutoffs that are defined in prospective observational studies using ROC analyses should be tested through prospective randomized studies to assess the safety, efficacy, and cost-effectiveness of customized approaches based on platelet function testing results. In general, various platelet function tests provide a high negative predictive values ${ }^{38}$ which in turn may allow for (1) a reduction in the number of days that patients with a preoperatively administered dual APT are off antiplatelet agents, as suggested by the current guidelines ${ }^{28,29}$ and for (2) reduction of procoagulant components transfusion rate. Such an approach would lead to the determination of the optimal safe period from discontinuation of APT to surgery for the individual patient and would minimize unnecessary transfusions. 
Again, such a tailored approach should be evaluated through prospective randomized clinical outcome evaluating trials.

As previously mentioned, prospective studies are the most suitable for assessment of bleeding risks and transfusion requirements. However, there are several retrospective studies addressing same issue. ${ }^{44,48,49,51}$ The major drawback of retrospective studies is the fact that evaluated platelet function testing was used in clinical decision-making process. Although such studies have often high number of patients from databases, it is obvious that observed associations are in certain degree distorted by the fact that platelet function tests were used in clinical decision-making process, thus probably creating bias in estimations of relationship between test results and both bleeding extent and transfusion requirements.

When discontinuing preoperative APT, one should be aware that some proportion of those patients is resistant to APT (have high degree of residual platelet reactivity despite appropriate treatment). Our working group recently found $31.3 \%$ of patients to be ASA resistant preoperatively. ${ }^{52}$ Therefore, ASA discontinuation in this subgroup of patients may lead to further increase of platelet reactivity and pose patients to proclivity to ischemic events. In addition to resistance to CLO therapy, increasingly administered antiplatelet drug in preoperative phase may be present in range between 25 and $60 \% .{ }^{53}$ Therefore, use of point-of-care platelet function analyzers may prevent not only bleeding events but also ischemic events by detecting those patients who experience high on-treatment platelet reactivity who might benefit from late or no APT discontinuation. Too early APT discontinuation may lead to onset of platelet hyperactivity ${ }^{54,55}$ and subsequently cause adverse ischemic events in preoperative phase. Finally, use of platelet function analyzers may provide dual benefit in terms of prevention of both bleeding and ischemic events.

Literature review revealed both positive and negative studies regarding association between platelet function analyzer findings and both bleeding outcomes and transfusion requirements. Heterogeneity of devices and variability in study settings hampers evidence and makes it somehow hard to pool the findings and make strong conclusions. First, there is no generally accepted definition of excessive bleeding and observed definitions vary widely across literature (-Table 1). Standardized definition of excessive bleeding is necessitating. We propose measurement of CTD in first 24 hours and the extent of CTD should be divided by patient's weight. Patients should be characterized as bleeders if their 24-hour CTD ( $\mathrm{mL} / \mathrm{kg})$ exceeds 75 th percentile of distribution in predefined sample of patients. Such a definition makes the most reliable correlation, and is not distorted to different anthropometric measures, perfusionistic, surgical, and anesthetic techniques. Such an approach dichotomizes bleeding outcome. The alternative approach would be to correlate platelet function test findings with CTD as expressed in the form of continuous variable. Any significant correlations should further be tested for accuracy via ROC analysis. ${ }^{43}$ In this way, it would be possible to reach the best sensitivity/ specificity ratio as well as positive and negative predictive values. Drawbacks of the CTD quantification were briefly discussed by Ti et al. ${ }^{9}$ One should be aware that blood loss measured as CTD consists of a mixture of fluids, including actual blood loss, serous drainage, and fluid left in the pleural cavities. Furthermore, bleeding is consisted of two types depending on the origin of bleeding: (1) bleeding due to hemostatic disorder including platelet dysfunction and (2) bleeding due to surgical issues.

Treatment options of hemorrhage in cardiac surgery are continuously evolving. Recently, Görlinger et al provided comprehensive insight into this issue. ${ }^{56}$ It is, however, obvious that appropriate hemostatic management in cardiac surgery patients should be consisted of bundle of protective strategies to prevent and treat excessive bleeding such as the following:

1. Preoperative bleeding risk stratification, based mainly on detection of patients who have weak platelet function, thus proclivity to excessive bleeding. This part is largely elaborated in the present review article.

2. Intraoperative transfusion management based on pointof-care hemostatic tests. There is evidence for efficacy of such a transfusion management based on tests for assessments of viscoelastic blood properties (ROTEM) and platelet function tests. ${ }^{47}$ Different treatment modalities are available depending on possibilities of each cardiac surgery center. We propose algorithm for perioperative hemostatic management that we use at our center (-Fig. 1). Such an algorithm provides a concept that may be applied generally and adjusted to different point-of-care hemostatic tests as well as different hemostatic treatment modalities available.

3. Surgical measures to achieve meticulous hemostasis.

Bleeding due to surgical issues should not be underestimated for two reasons: (1) it certainly affects the extent of CTD which was found to be independently associated with adverse outcomes after cardiac surgery ${ }^{2,3}$ and (2) more than $70 \%$ of patients undergoing reexploration for excessive bleeding have had bleeding vessel identified (surgical cause of bleeding). ${ }^{4}$ Surgeon performance may contribute significantly to such a complication. Biancari et al hypothesized that individual surgeon's performance may contribute significantly to bleeding outcome. ${ }^{4}$ Retrospective study on 2001 patients reported reexploration for bleeding rate of $5.3 \%{ }^{4}$ of more importance rates of reexploration varied between 1.4 and $11.7 \%$ ( $p<0.0001$ ) according to different surgeon's performing hemostasis. ${ }^{4}$ Furthermore, variable of individual surgeon was found to be independent predictor for reexploration for excessive bleeding, as well as for CTD of $\geq 1,600 \mathrm{~mL}{ }^{4} \mathrm{Kim}$ et al demonstrated that individual surgeon was found to be strongest factor associated with transfusion outcome in patients undergoing CABG with recent CLO exposure. ${ }^{57}$ Therefore, meticulous surgical technique must be cornerstone on appropriate hemostatic management because individual surgeon's skills may be important as much as the preoperative APT discontinuation management based on platelet function testing. Moreover, Loor et $\mathrm{al}^{58}$ developed and implemented a formal operative checklist to reduce 


\begin{tabular}{|c|c|c|c|c|c|}
\hline 施 & 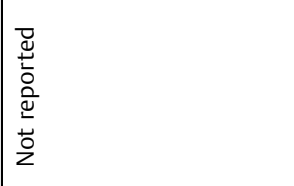 & 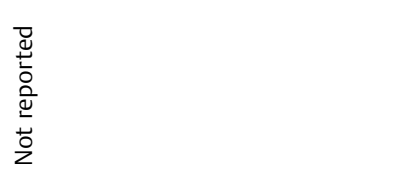 & 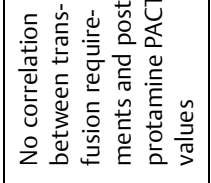 & 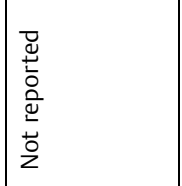 & 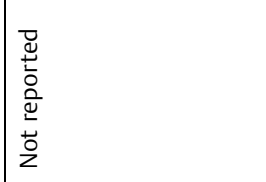 \\
\hline 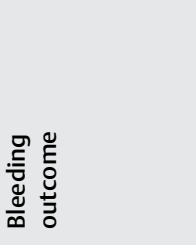 & 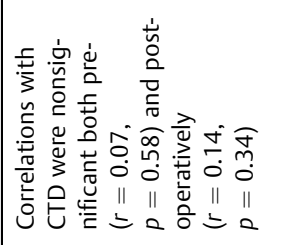 & 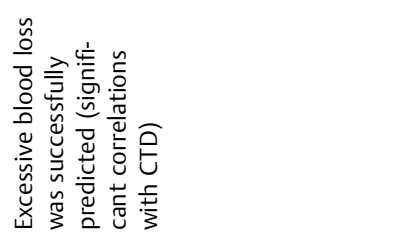 & & 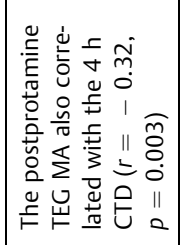 & 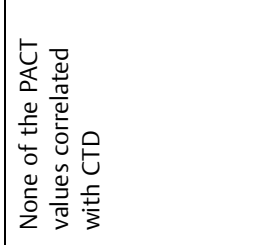 \\
\hline 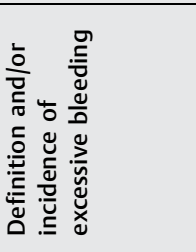 & 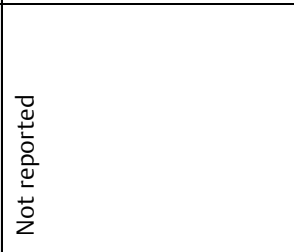 & 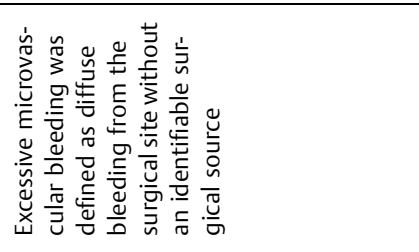 & \multicolumn{2}{|l|}{ 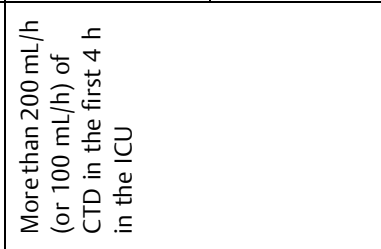 } & 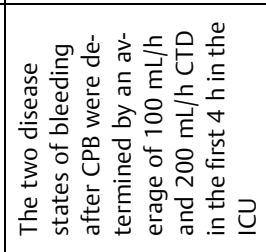 \\
\hline 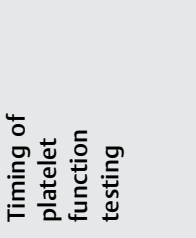 & 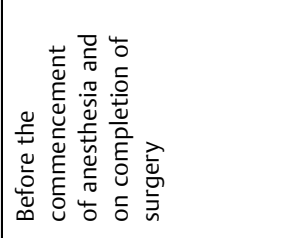 & 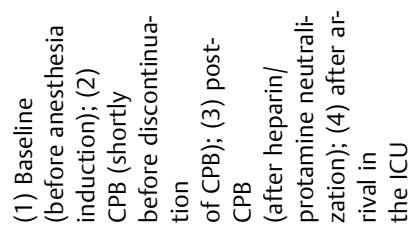 & \multicolumn{2}{|c|}{ 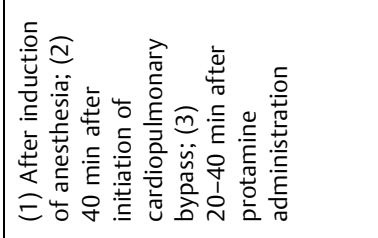 } & 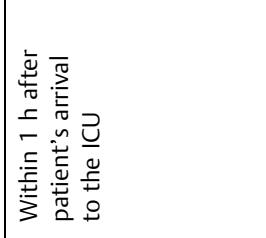 \\
\hline 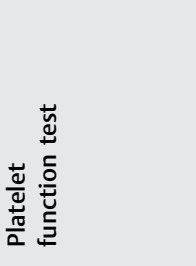 & 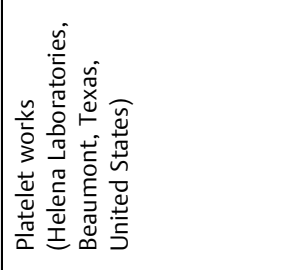 & 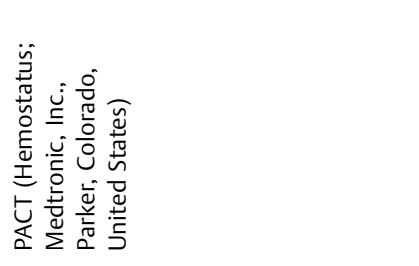 & 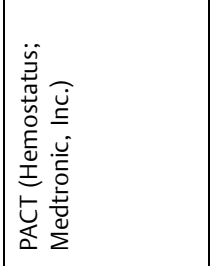 & 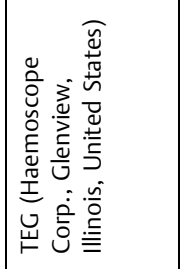 & 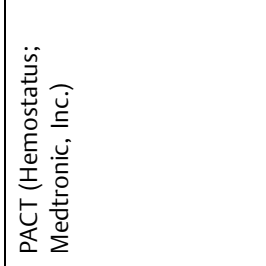 \\
\hline & 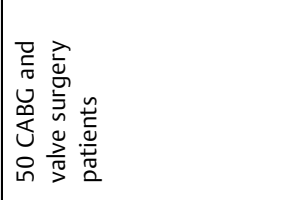 & 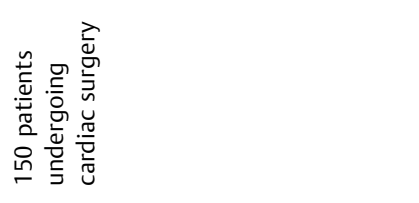 & \multicolumn{2}{|l|}{ 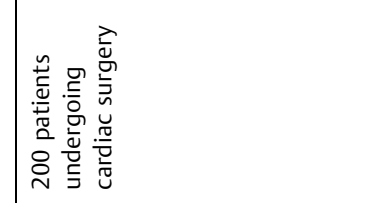 } & 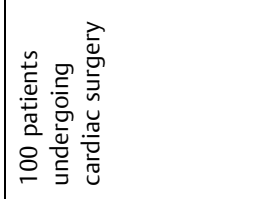 \\
\hline 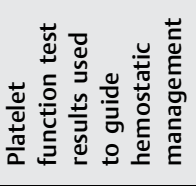 & $\stackrel{0}{2}$ & $\stackrel{0}{2}$ & \multicolumn{2}{|l|}{ z } & z \\
\hline 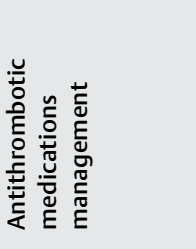 & 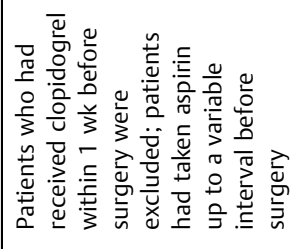 & 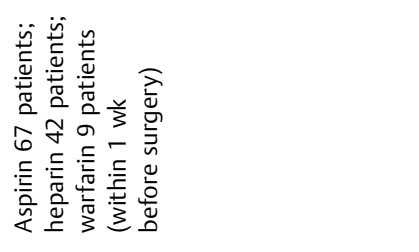 & \multicolumn{2}{|l|}{ 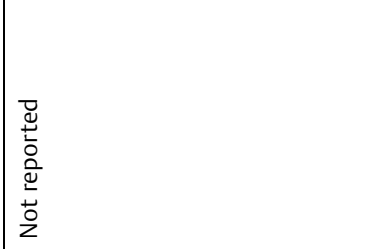 } & 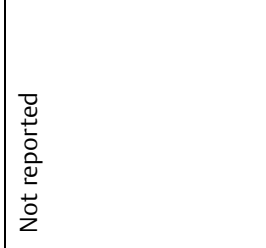 \\
\hline 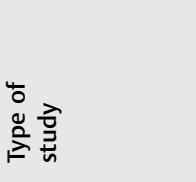 & 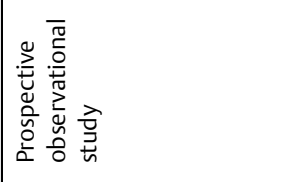 & 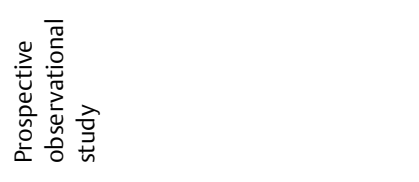 & \multicolumn{2}{|l|}{ 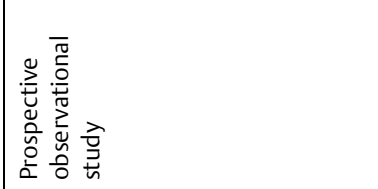 } & 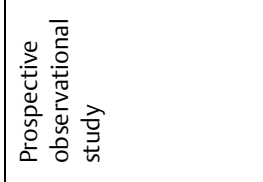 \\
\hline 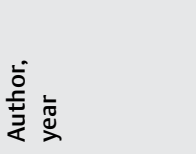 & 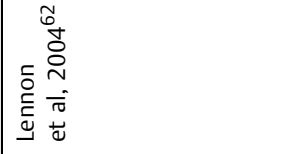 & 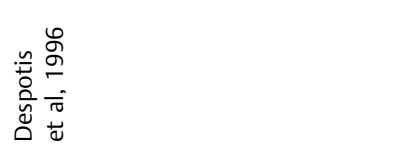 & \multicolumn{2}{|l|}{ 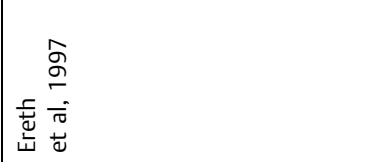 } & 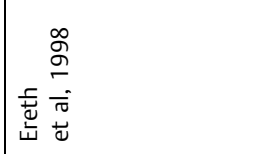 \\
\hline
\end{tabular}




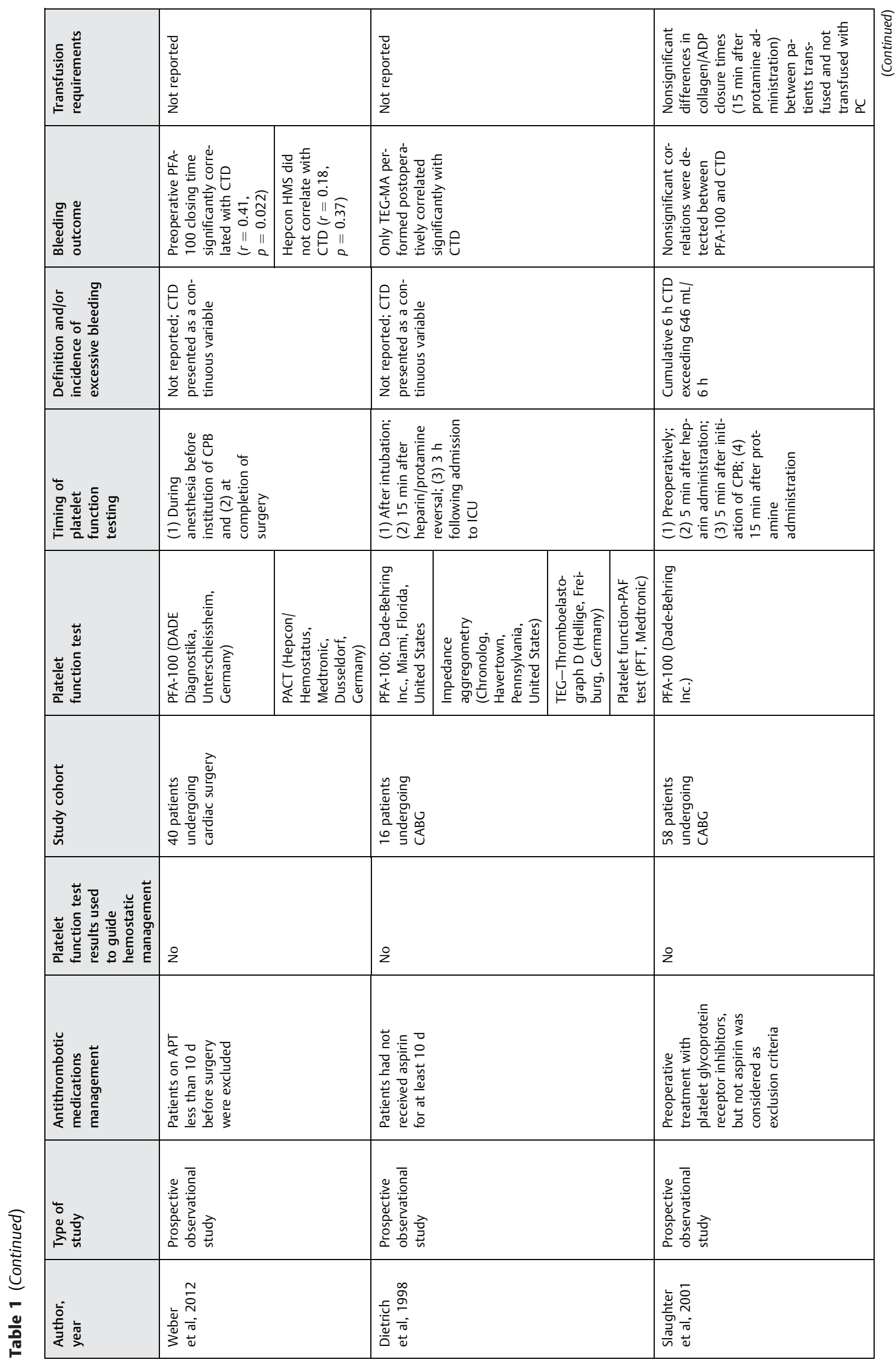


12 Predictive Value of Platelet Function Point-of-Care Tests Petricevic et al.

\begin{tabular}{|c|c|c|c|c|c|c|}
\hline 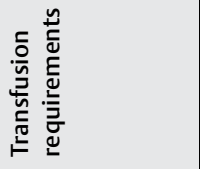 & \multicolumn{2}{|l|}{ 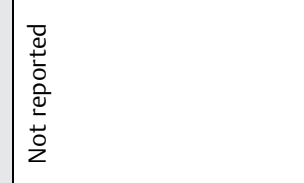 } & 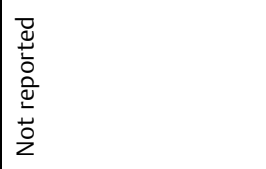 & 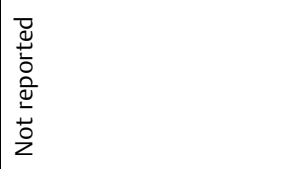 & \multicolumn{2}{|l|}{ 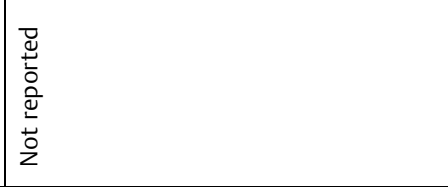 } \\
\hline 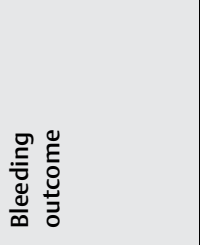 & \multicolumn{2}{|l|}{ 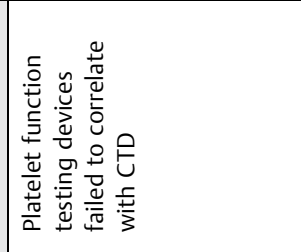 } & 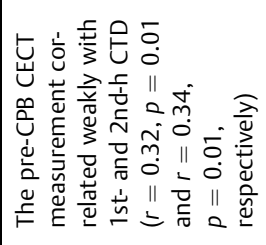 & 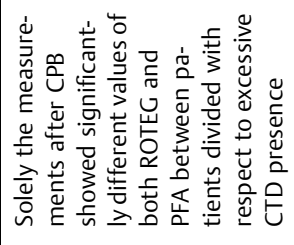 & \multicolumn{2}{|c|}{ 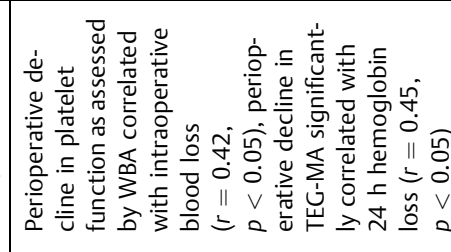 } \\
\hline 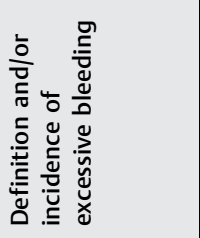 & \multicolumn{2}{|l|}{ 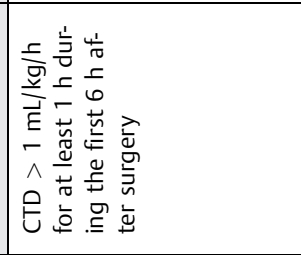 } & 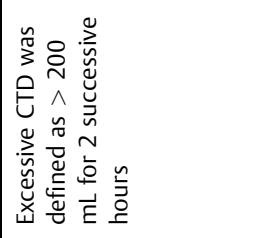 & 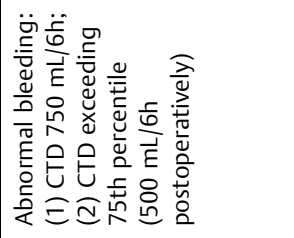 & \multicolumn{2}{|l|}{ 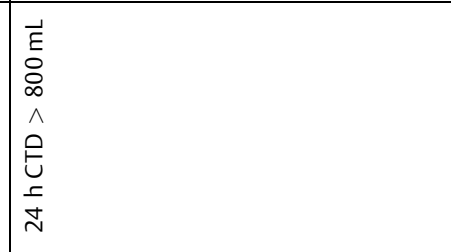 } \\
\hline 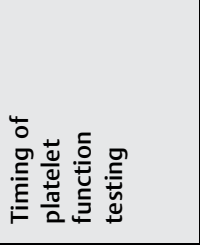 & \multicolumn{2}{|l|}{ 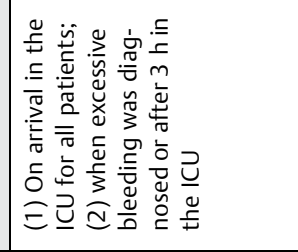 } & 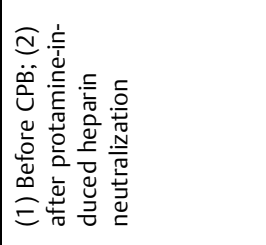 & 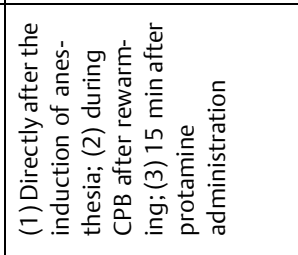 & \multicolumn{2}{|c|}{ 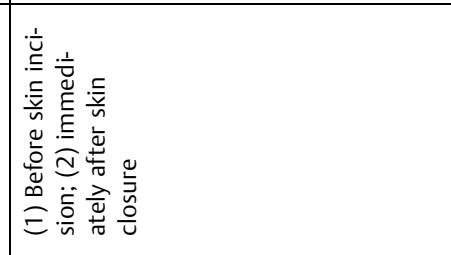 } \\
\hline 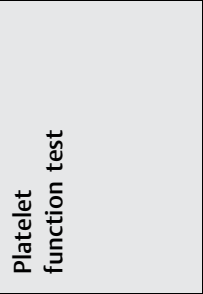 & 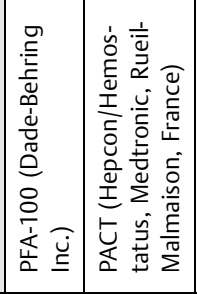 & 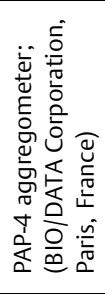 & 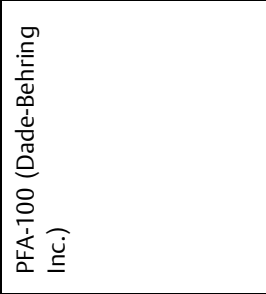 & 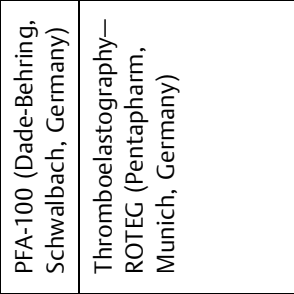 & 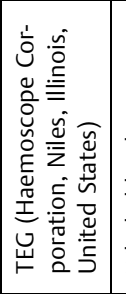 & 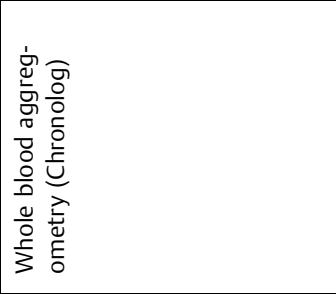 \\
\hline & \multicolumn{2}{|l|}{ 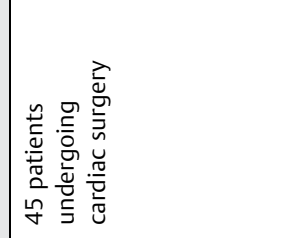 } & 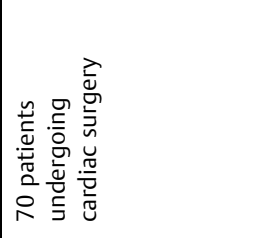 & 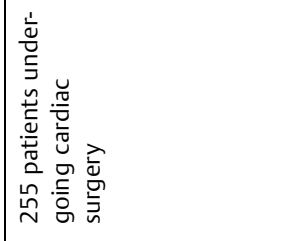 & \multicolumn{2}{|l|}{ 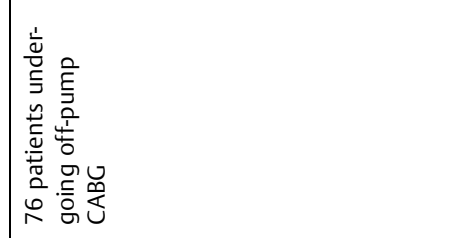 } \\
\hline 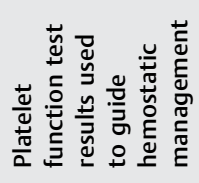 & \multicolumn{2}{|l|}{ 2 } & 20 & 2o & \multicolumn{2}{|l|}{ 2o } \\
\hline 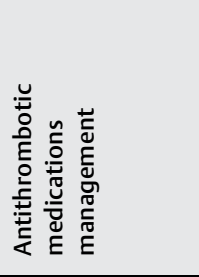 & \multicolumn{2}{|c|}{ 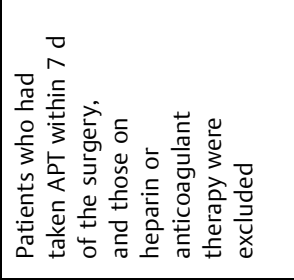 } & 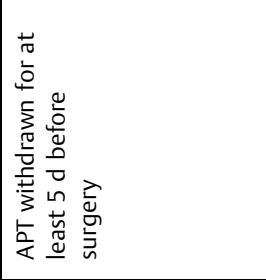 & 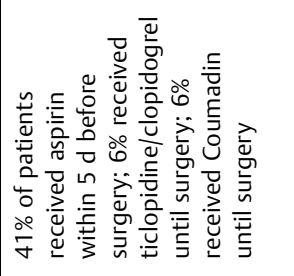 & \multicolumn{2}{|c|}{ 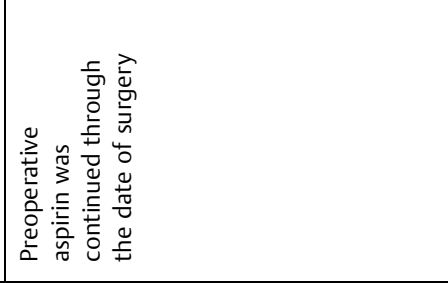 } \\
\hline 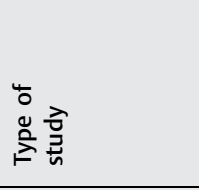 & \multicolumn{2}{|l|}{ 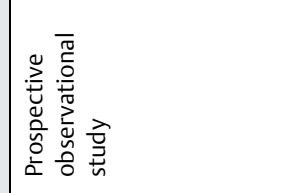 } & 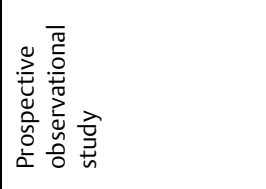 & 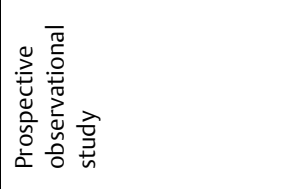 & \multicolumn{2}{|l|}{ 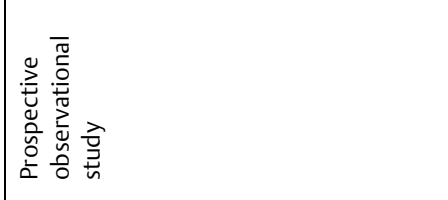 } \\
\hline 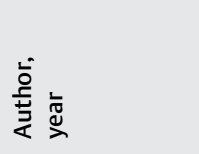 & \multicolumn{2}{|l|}{ 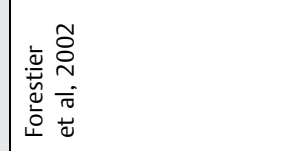 } & 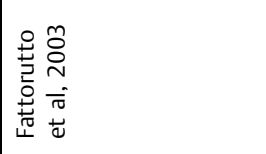 & 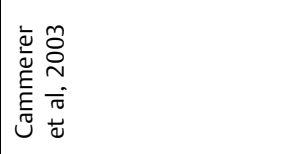 & \multicolumn{2}{|l|}{ 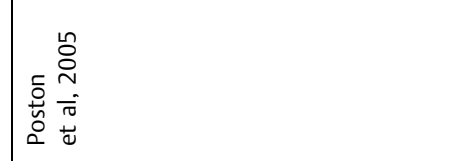 } \\
\hline
\end{tabular}




\begin{tabular}{|c|c|c|c|c|}
\hline 总 & 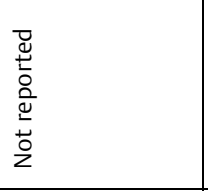 & 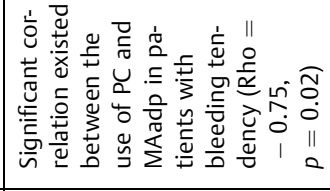 & 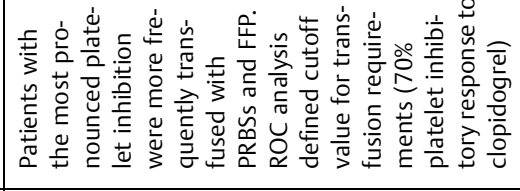 & 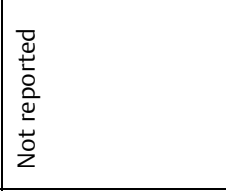 \\
\hline 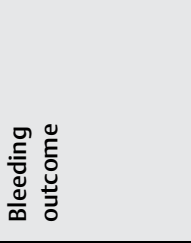 & 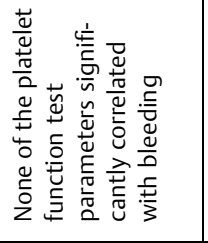 & 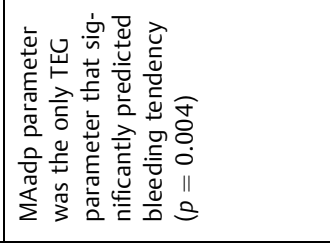 & 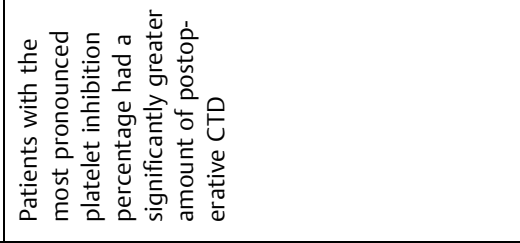 & 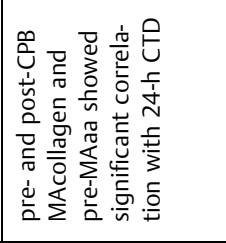 \\
\hline 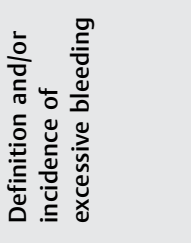 & 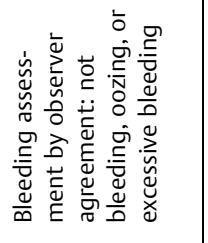 & 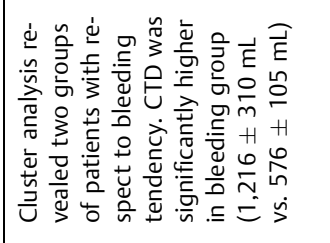 & 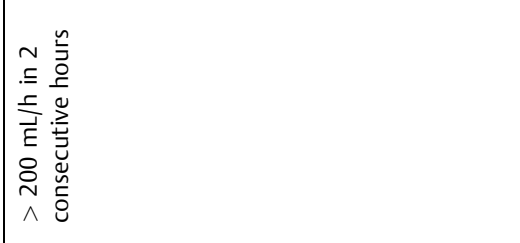 & 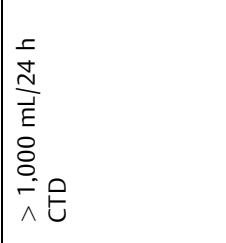 \\
\hline 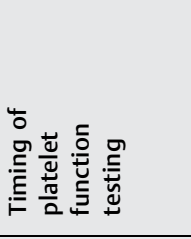 & 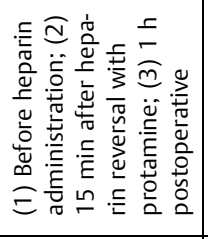 & 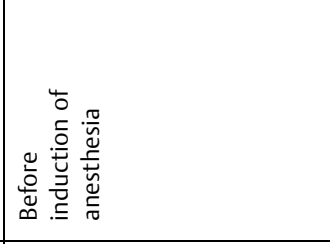 & 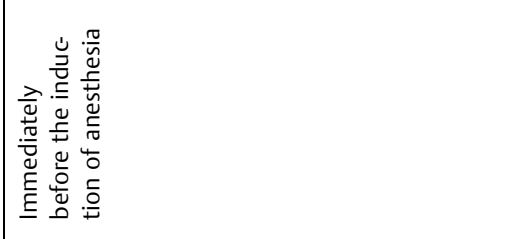 & 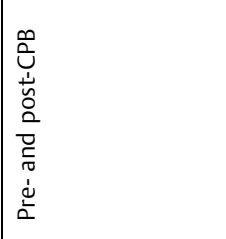 \\
\hline 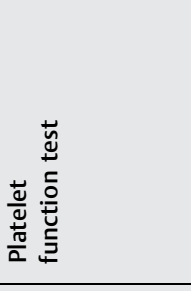 & 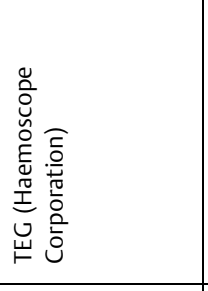 & 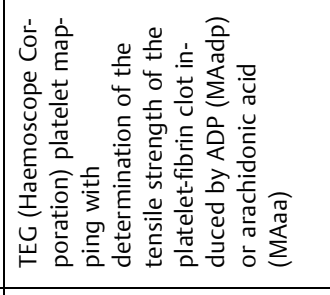 & 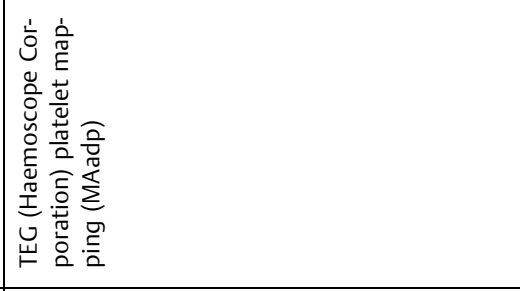 & 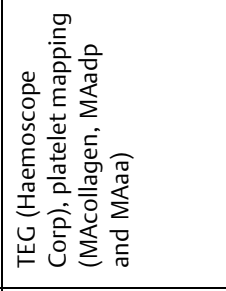 \\
\hline 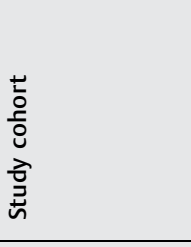 & 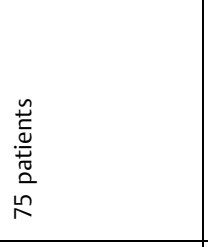 & 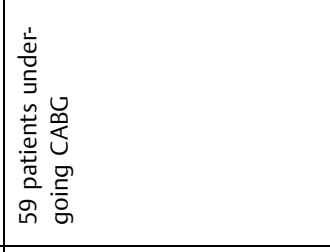 & 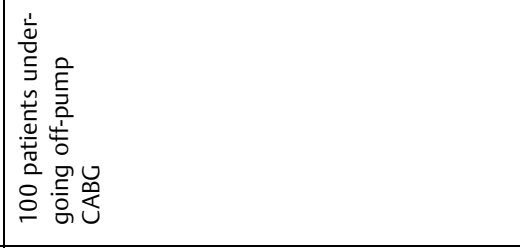 & 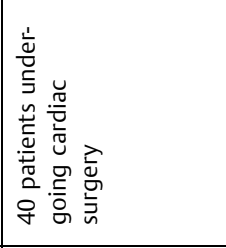 \\
\hline 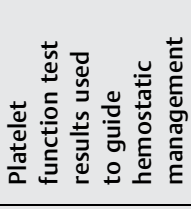 & $\stackrel{2}{2}$ & $\stackrel{2}{2}$ & z & 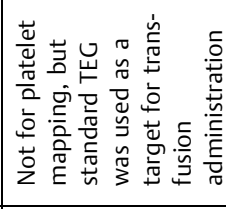 \\
\hline 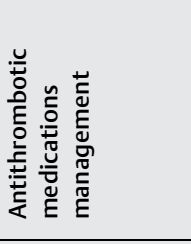 & 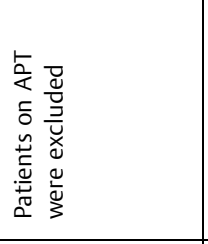 & 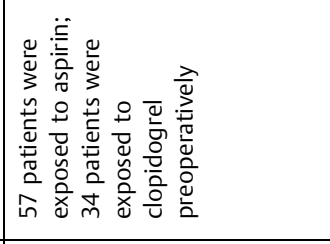 & 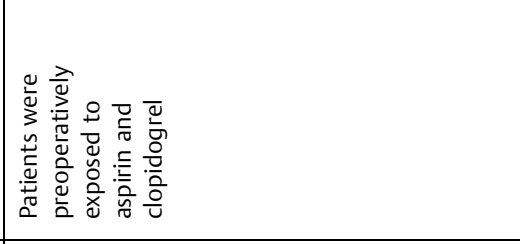 & 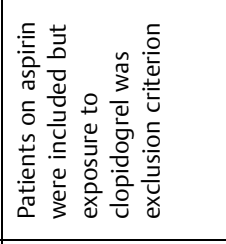 \\
\hline 悥 & 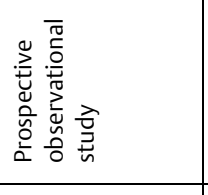 & 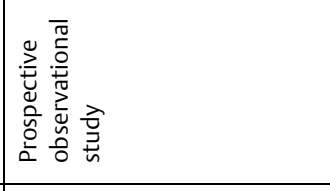 & 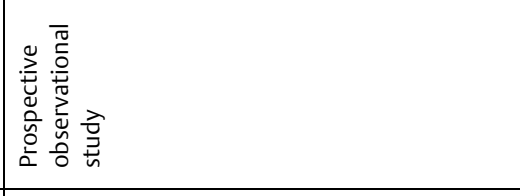 & 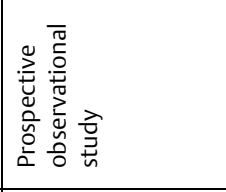 \\
\hline 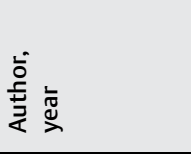 & 总 & 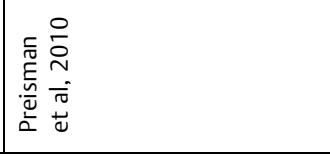 & 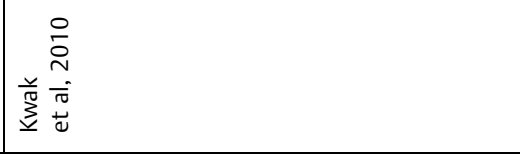 & 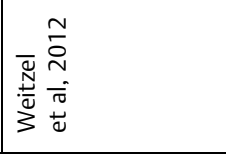 \\
\hline
\end{tabular}


14 Predictive Value of Platelet Function Point-of-Care Tests Petricevic et al.

\begin{tabular}{|c|c|c|c|}
\hline 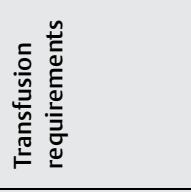 & 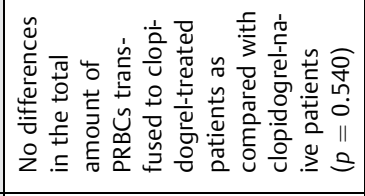 & 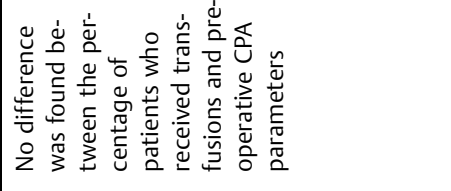 & 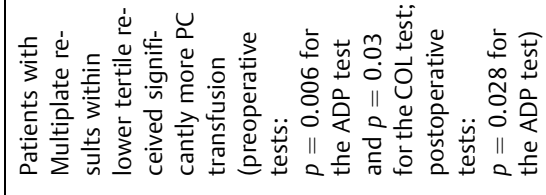 \\
\hline 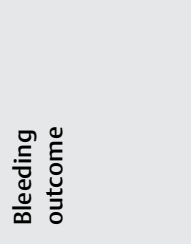 & 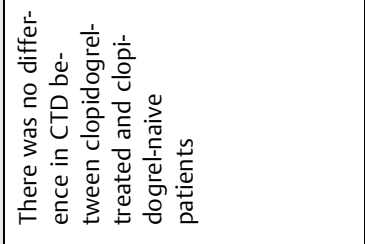 & 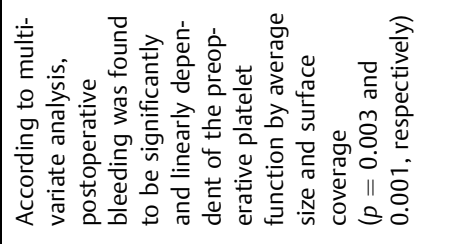 & 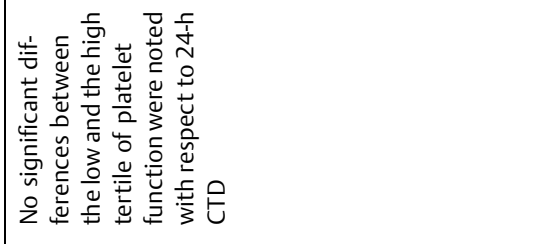 \\
\hline 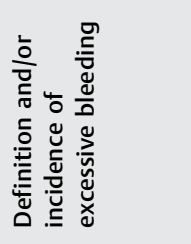 & 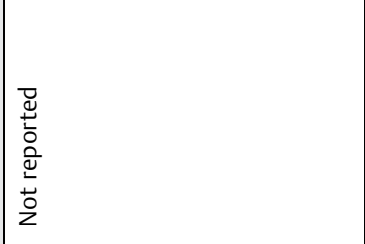 & 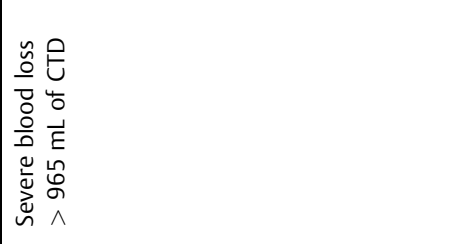 & 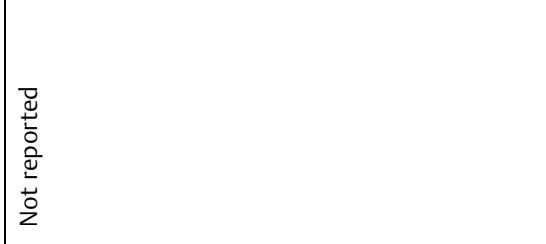 \\
\hline 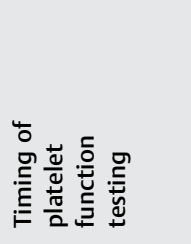 & 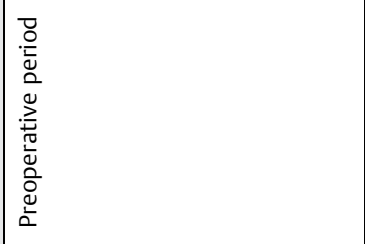 & 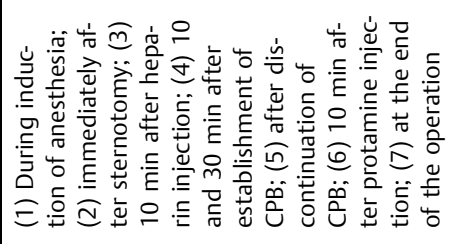 & 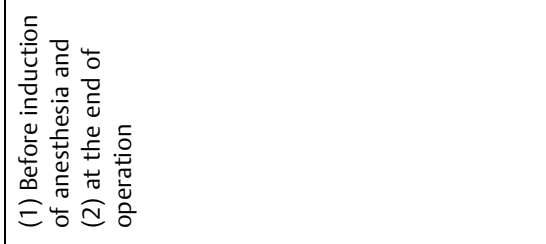 \\
\hline 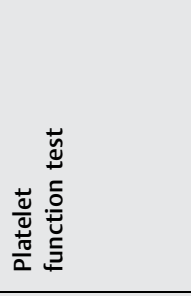 & 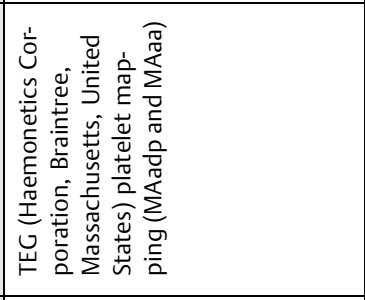 & 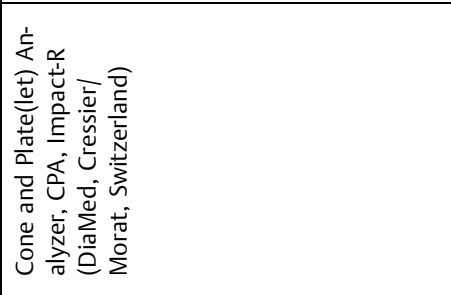 & 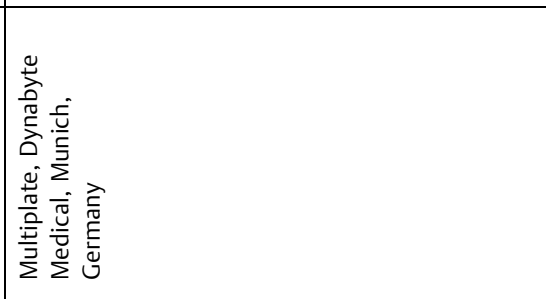 \\
\hline 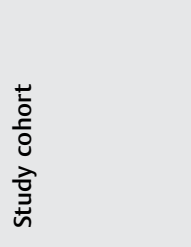 & 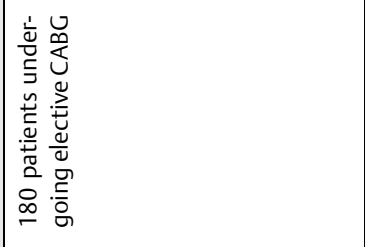 & 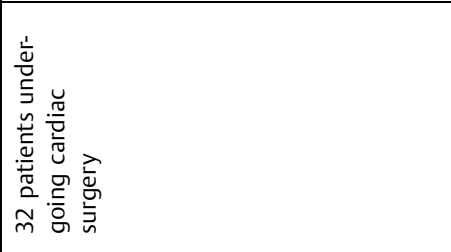 & 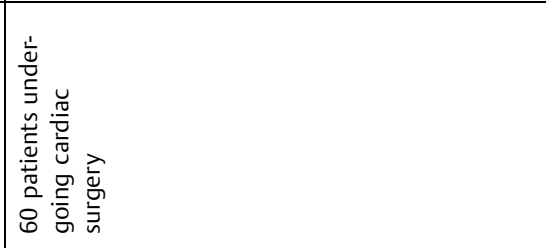 \\
\hline 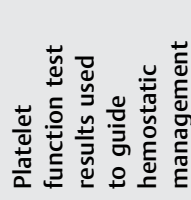 & 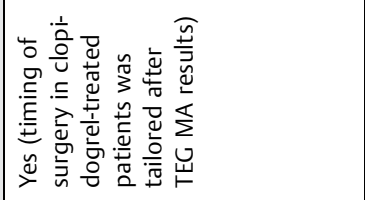 & q & $\stackrel{2}{2}$ \\
\hline 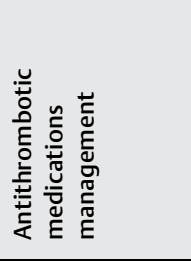 & 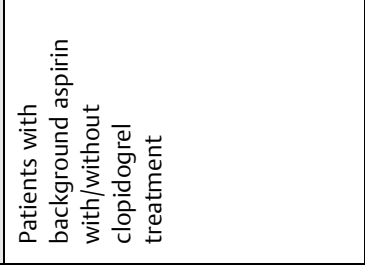 & 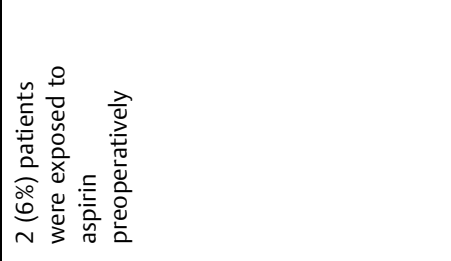 & 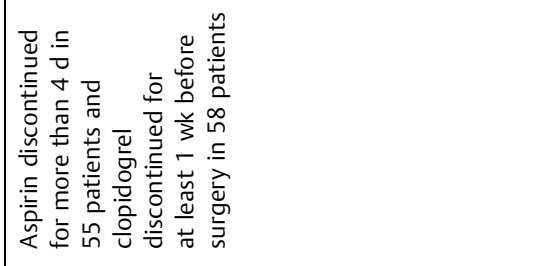 \\
\hline 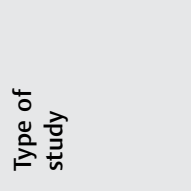 & 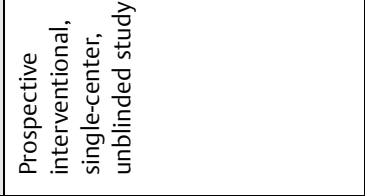 & 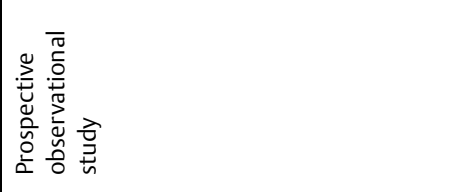 & 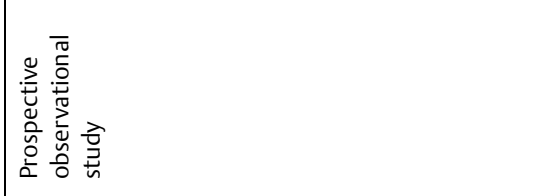 \\
\hline 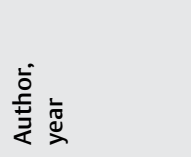 & 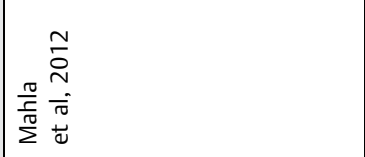 & 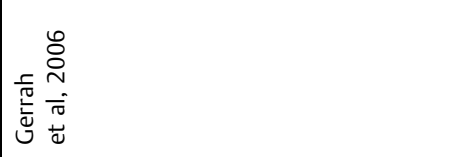 & 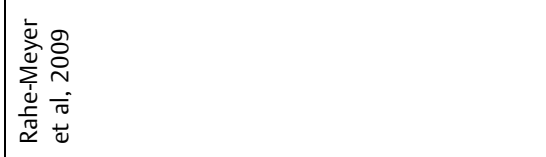 \\
\hline
\end{tabular}




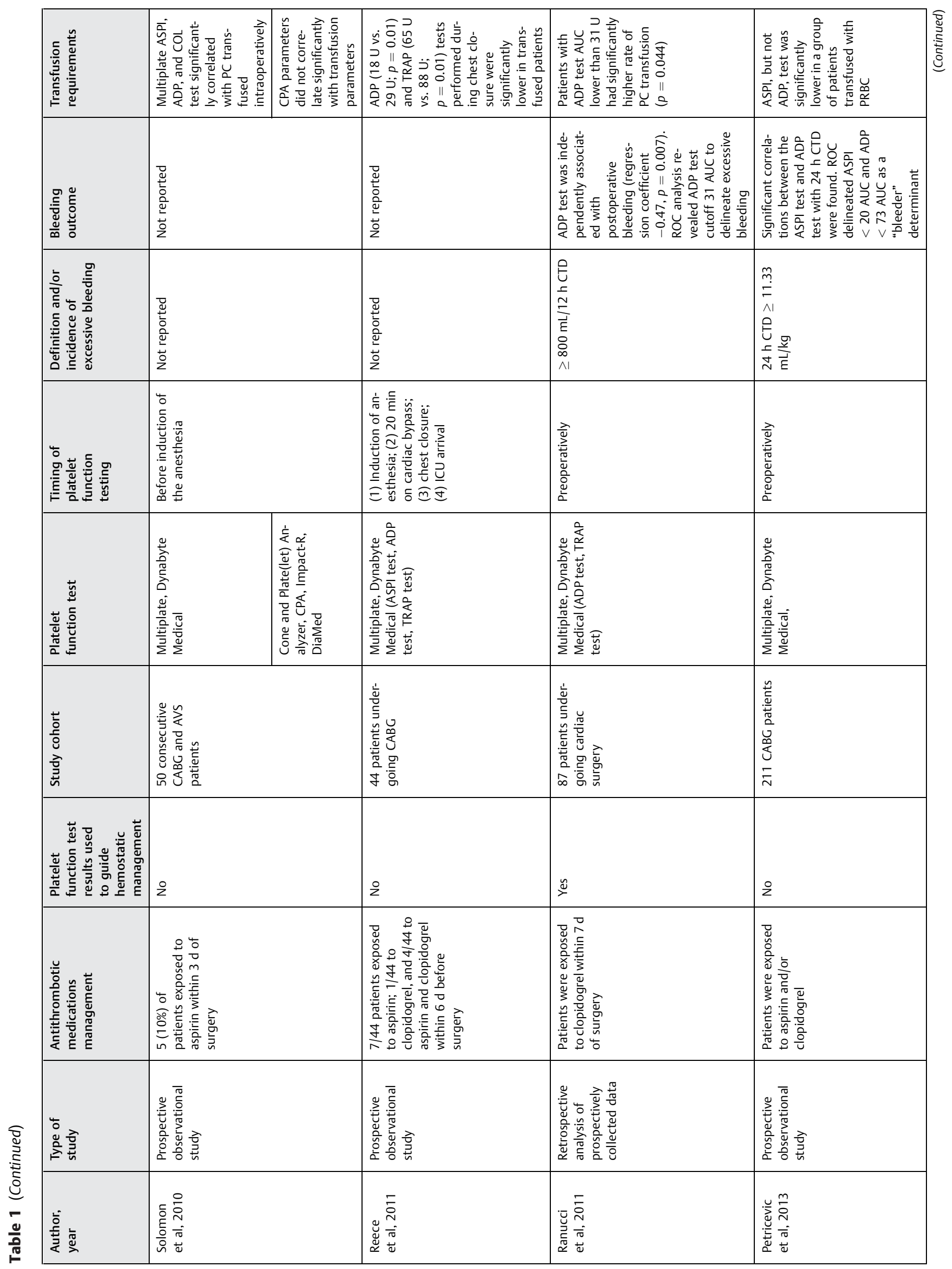




\begin{tabular}{|c|c|c|c|c|c|}
\hline 它 & 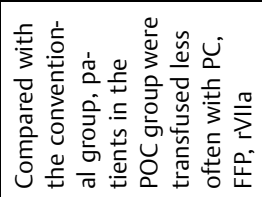 & 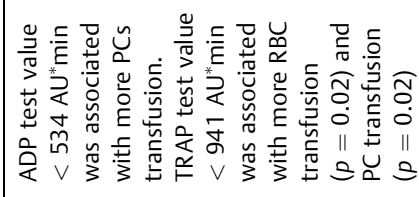 & \multicolumn{2}{|l|}{ 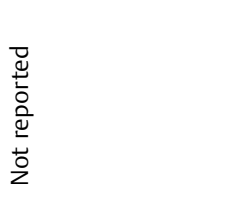 } & 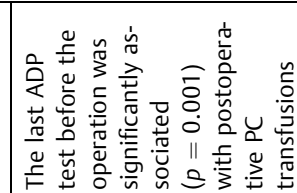 \\
\hline 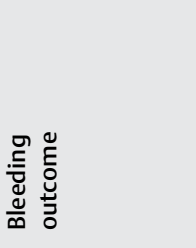 & 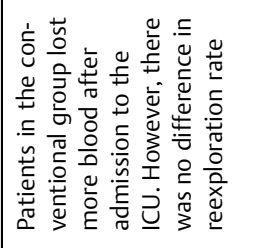 & 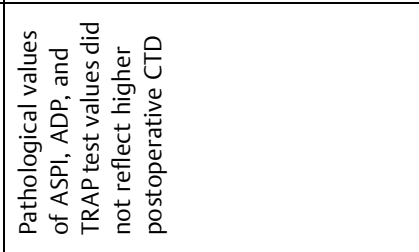 & 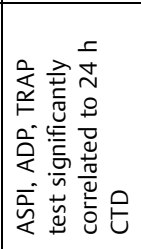 & 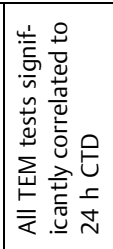 & 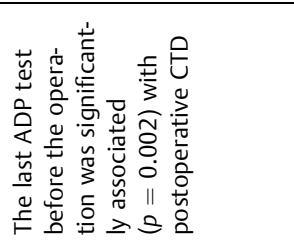 \\
\hline 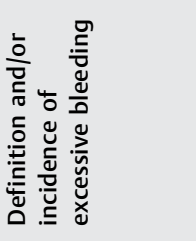 & 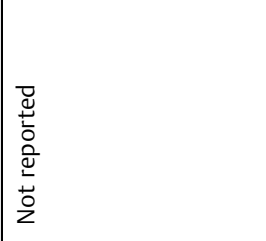 & 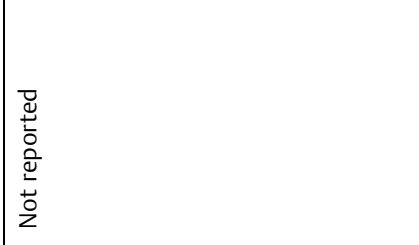 & 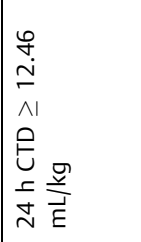 & & 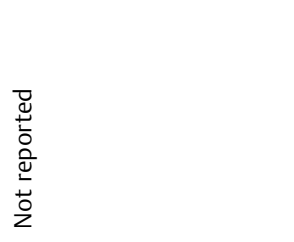 \\
\hline 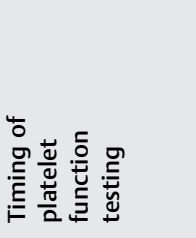 & 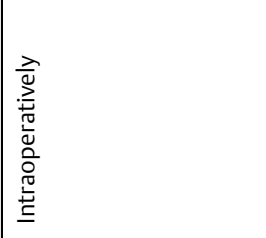 & 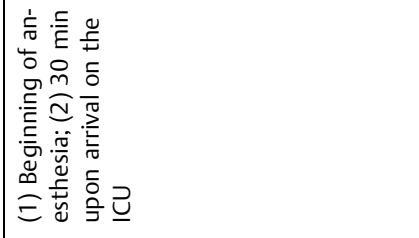 & 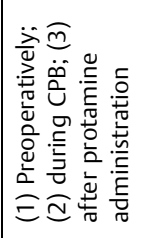 & & 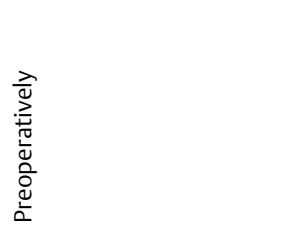 \\
\hline 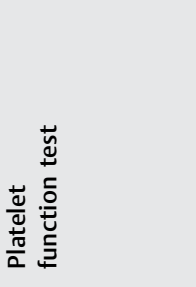 & 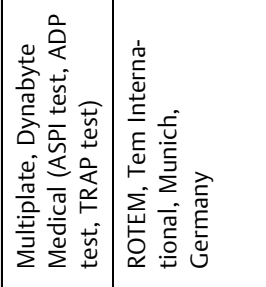 & 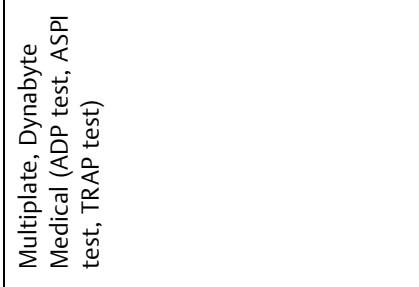 & 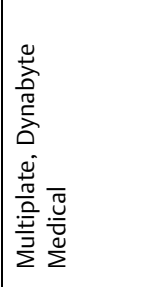 & 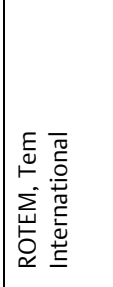 & 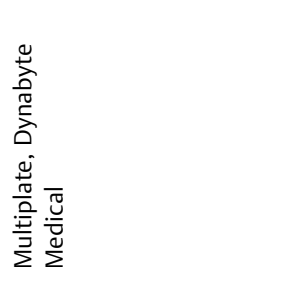 \\
\hline 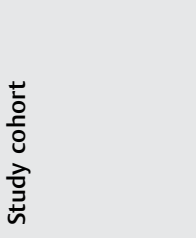 & 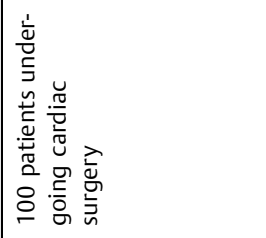 & 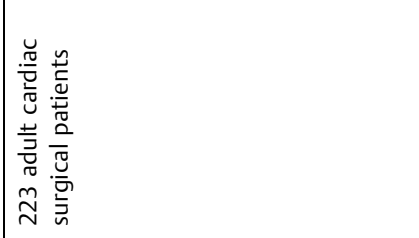 & 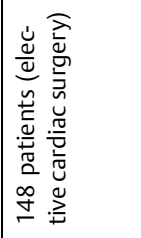 & & 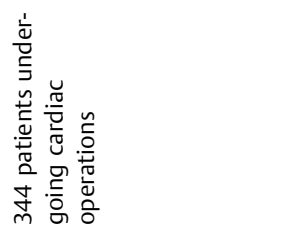 \\
\hline 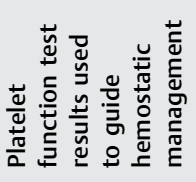 & $\stackrel{y}{\nu}$ & $\stackrel{y}{\nu}$ & 2o & & $\stackrel{\check{\nu}}{\check{\nu}}$ \\
\hline 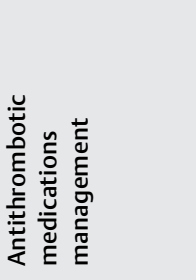 & 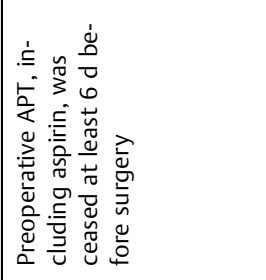 & 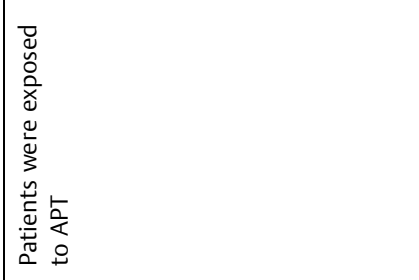 & 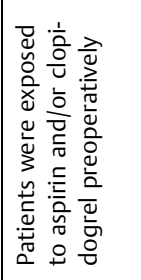 & & 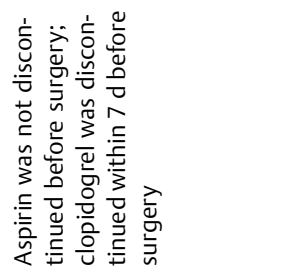 \\
\hline 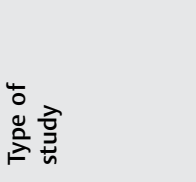 & 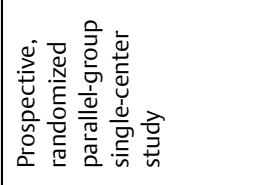 & 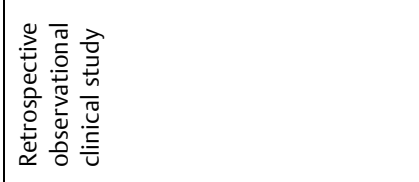 & 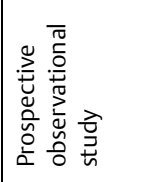 & & 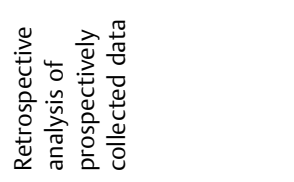 \\
\hline 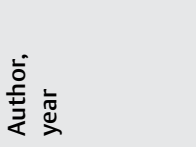 & 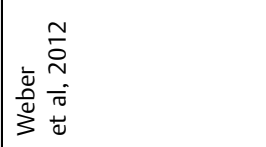 & 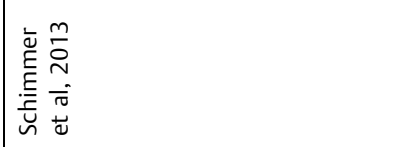 & 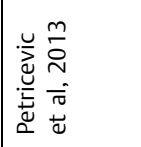 & & 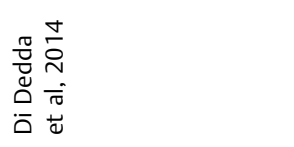 \\
\hline
\end{tabular}



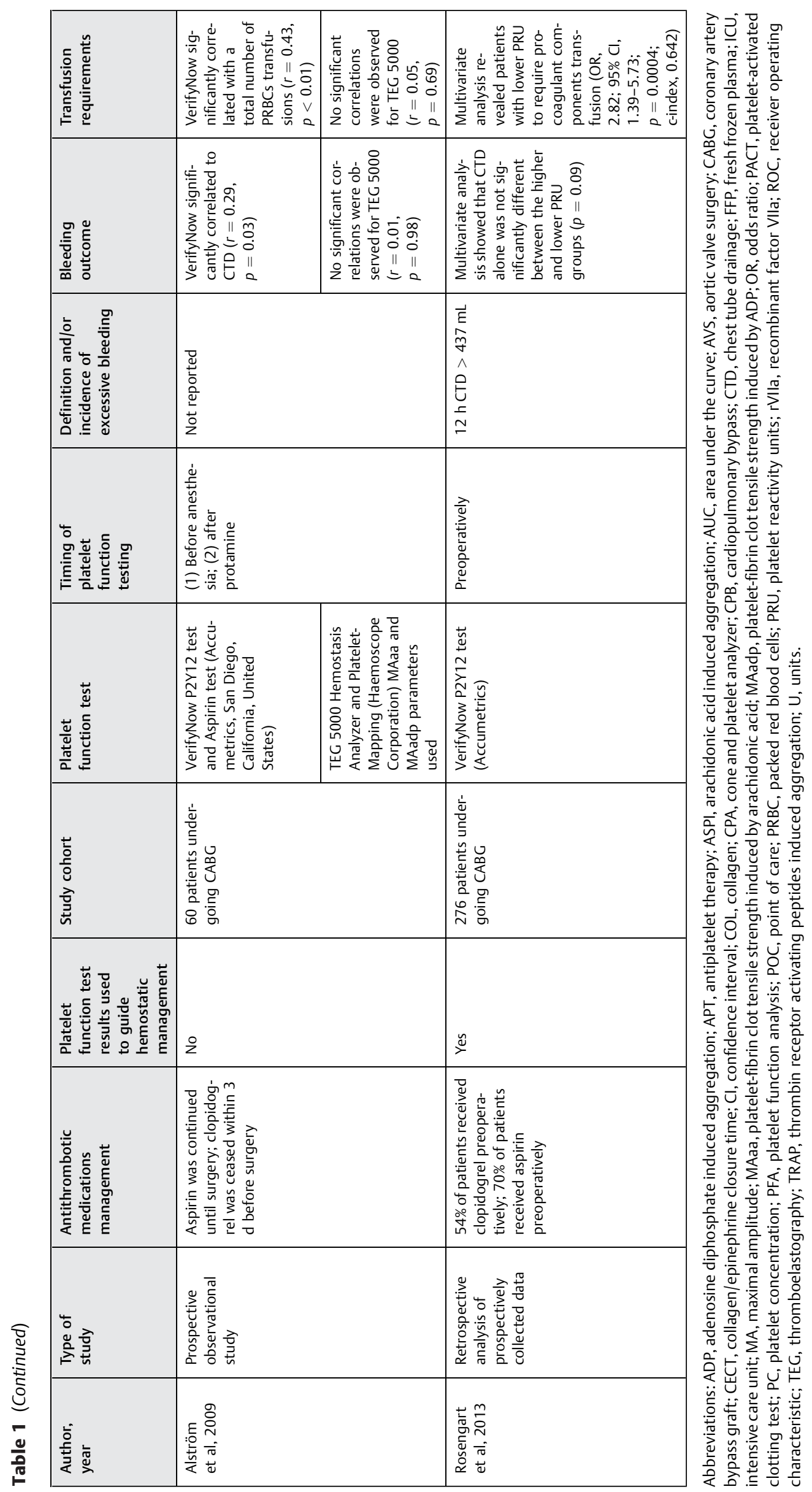


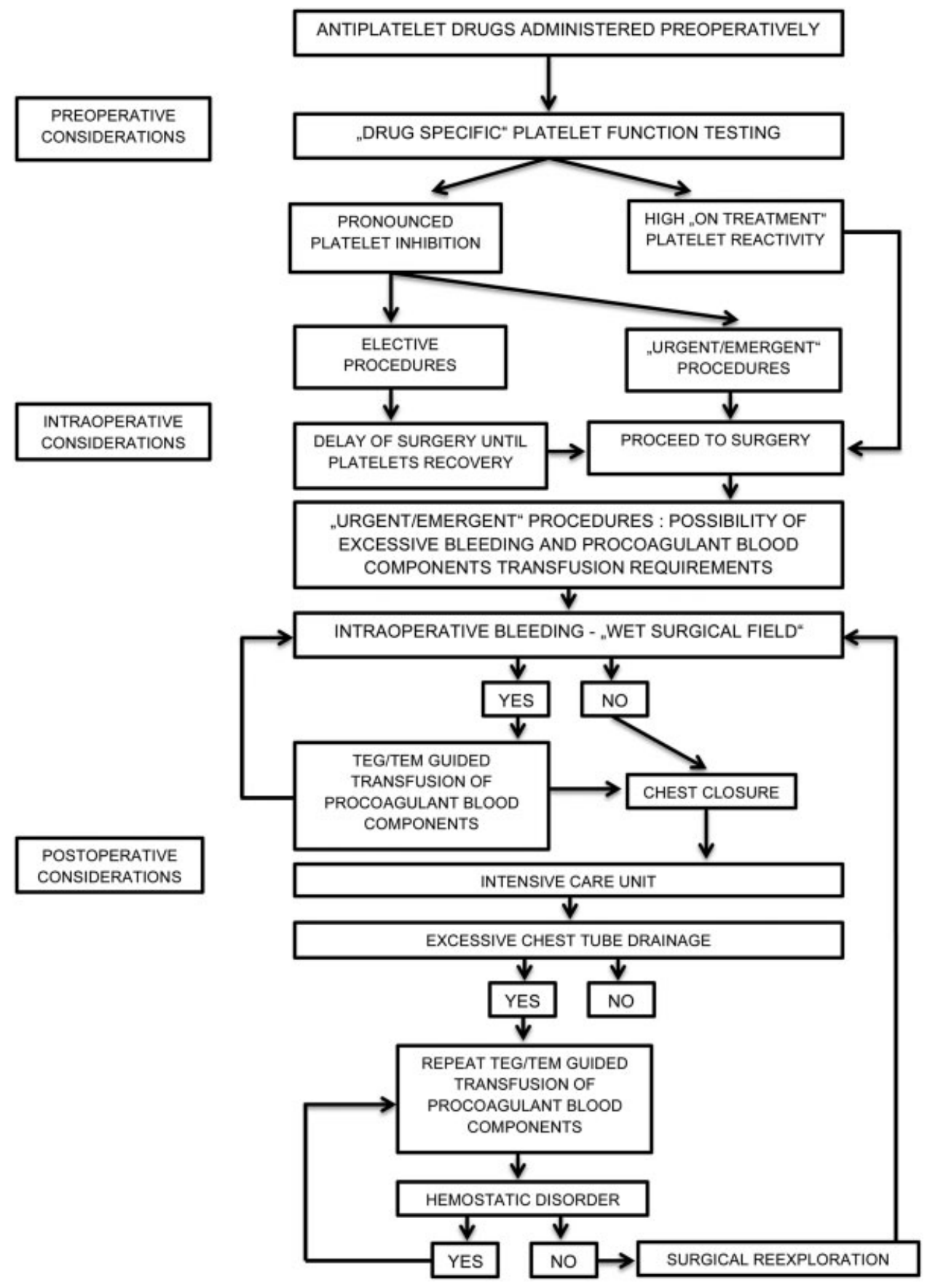

Fig. 1 Perioperative hemostatic management algorithm. TEG/TEM: thromboelastography/thromboelastometry.

technical reasons for postoperative bleeding..$^{58}$ Easily performed hemostasis checklist based on the most common sites of bleeding was developed. ${ }^{58}$ Implementation of such bleeding checklist resulted in increased number of cases operated on between consecutive reoperations. ${ }^{58}$ Therefore, to optimize bleeding outcomes, meticulous surgical technique along with checklist for bleeding should be inextricably included to perioperative APT administration management targeted after platelet function testing.

In conclusion, appropriate perioperative hemostatic management should inevitably include bedside suitable point-ofcare devices and hemostatic management should be based on goal-directed hemostatic therapy, the so-called theranostic approach. ${ }^{44,47,56,59-61}$ Such an approach may reduce the rate of reexploration for bleeding from up to 75 to $20 \%$, ${ }^{4,47}$ as recently described by Weber et al through prospective randomized trial. ${ }^{47}$ Point-of-care guided hemostatic algorithm may reduce the rate of reexplorations due to hemostatic disorder and direct reexploration surgery dominantly to surgical cause of bleeding. The reduction of unnecessary as well as inefficient surgical reexplorations rate certainly may improve clinical outcome. There are a large number of studies dealing with prediction and prevention of excessive bleeding in cardiac surgery patients. Platelet function analyzers should 
definitely be included in modern hemostatic management with aim to frame safety therapeutic window (between bleeding and ischemic events). We suggest use of platelet function testing in perioperative setting of cardiac surgery patients. However, when using such devices, one should be aware of platelet function testing limitations in estimating risk for excessive bleeding and transfusion requirements. Such devices may not always provide accurate prediction of bleeding which can be easily explained by the fact that bleeding is consisted of both surgical bleeding and bleeding due to hemostatic disorder of which platelet function is only one part. In process of decision making based on point-of-care hemostatic tests, one should account with the possibility of excessive bleeding despite normal values of hemostatic tests. The reason for such a phenomenon may be the fact that surgical hemostasis is not always as good as it should be, in particular in teaching hospitals. Meticulous surgical hemostasis must be performed to exclude surgical cause of bleeding. Procoagulant blood component administration should be targeted after both platelet function assay results and TEM/ TEG results. Antifibrinolytics as well as DDAVP should be considered in cases where weak platelet function or high fibrinolysis index is detected using point-of-care devices. Multicausality in hemostatic disorders requires multidisciplinary and comprehensive approach in treatment. "Onesize-fits-for-all" approach in hemostatic management seems to be obsolete, and shift toward personalized approach using bedside-suitable point-of-care devices in bleeding and transfusion requirements optimization is certainly required.

\section{References}

1 Whitlock R, Crowther MA, Ng HJ. Bleeding in cardiac surgery: its prevention and treatment-an evidence-based review. Crit Care Clin 2005;21(3):589-610

2 Christensen MC, Dziewior F, Kempel A, von Heymann C. Increased chest tube drainage is independently associated with adverse outcome after cardiac surgery. J Cardiothorac Vasc Anesth 2012; 26(1):46-51

3 Dixon B, Santamaria JD, Reid D, et al. The association of blood transfusion with mortality after cardiac surgery: cause or confounding? (CME). Transfusion 2013;53(1):19-27

4 Biancari F, Mikkola R, Heikkinen J, Lahtinen J, Kettunen U, Juvonen T. Individual surgeon's impact on the risk of re-exploration for excessive bleeding after coronary artery bypass surgery. J Cardiothorac Vasc Anesth 2012;26(4):550-556

5 O'Donnell CJ, Larson MG, Feng D, et al; Framingham Heart Study. Genetic and environmental contributions to platelet aggregation: the Framingham heart study. Circulation 2001;103(25):3051-3056

6 Ben-Dor I, Kleiman NS, Lev E. Assessment, mechanisms, and clinical implication of variability in platelet response to aspirin and clopidogrel therapy. Am J Cardiol 2009;104(2):227-233

7 Mehta RH, Roe MT, Mulgund J, et al. Acute clopidogrel use and outcomes in patients with non-ST-segment elevation acute coronary syndromes undergoing coronary artery bypass surgery. J Am Coll Cardiol 2006;48(2):281-286

8 Spiess BD, Tuman KJ, McCarthy RJ, DeLaria GA, Schillo R, Ivankovich AD. Thromboelastography as an indicator of post-cardiopulmonary bypass coagulopathies. J Clin Monit 1987;3(1):25-30

9 Ti LK, Cheong KF, Chen FG. Prediction of excessive bleeding after coronary artery bypass graft surgery: the influence of timing and heparinase on thromboelastography. J Cardiothorac Vasc Anesth 2002;16(5):545-550

10 Velik-Salchner C, Maier S, Innerhofer P, et al. An assessment of cardiopulmonary bypass-induced changes in platelet function using whole blood and classical light transmission aggregometry: the results of a pilot study. Anesth Analg 2009;108(6):1747-1754

11 Fitchett D, Mazer CD, Eikelboom J, Verma S. Antiplatelet therapy and cardiac surgery: review of recent evidence and clinical implications. Can J Cardiol 2013;29(9):1042-1047

12 Smith PK, Goodnough LT, Levy JH, et al. Mortality benefit with prasugrel in the TRITON-TIMI 38 coronary artery bypass grafting cohort: risk-adjusted retrospective data analysis. J Am Coll Cardiol 2012;60(5):388-396

13 Jernberg T, Payne CD, Winters KJ, et al. Prasugrel achieves greater inhibition of platelet aggregation and a lower rate of non-responders compared with clopidogrel in aspirin-treated patients with stable coronary artery disease. Eur Heart J 2006;27(10): 1166-1173

14 Bhatt DL, Stone GW, Mahaffey KW, et al; CHAMPION PHOENIX Investigators. Effect of platelet inhibition with cangrelor during PCI on ischemic events. N Engl J Med 2013;368(14):1303-1313

15 Angiolillo DJ, Firstenberg MS, Price MJ, et al; BRIDGE Investigators. Bridging antiplatelet therapy with cangrelor in patients undergoing cardiac surgery: a randomized controlled trial. JAMA 2012; 307(3):265-274

16 Casutt M, Konrad C, Schuepfer G. Effect of rivaroxaban on blood coagulation using the viscoelastic coagulation test ROTEM ${ }^{\mathrm{TM}}$. Anaesthesist 2012;61(11):948-953

17 Nascimento B, Rizoli S, Rubenfeld G, Lin Y, Callum J, Tien HC. Design and preliminary results of a pilot randomized controlled trial on a $1: 1: 1$ transfusion strategy: the trauma formula-driven versus laboratory-guided study. J Trauma 2011;71(5, Suppl 1):S418-S426

18 Paparella D, Brister SJ, Buchanan MR. Coagulation disorders of cardiopulmonary bypass: a review. Intensive Care Med 2004; 30(10):1873-1881

19 Jackson GN, Ashpole KJ, Yentis SM. The TEG vs the ROTEM thromboelastography/thromboelastometry systems. Anaesthesia 2009;64(2):212-215

20 Nielsen VG. A comparison of the Thrombelastograph and the ROTEM. Blood Coagul Fibrinolysis 2007;18(3):247-252

21 Seidel H, Rahman MM, Scharf RE. Monitoring of antiplatelet therapy. Current limitations, challenges, and perspectives. Hamostaseologie 2011;31(1):41-51

22 Poston R, Gu J, Manchio J, et al. Platelet function tests predict bleeding and thrombotic events after off-pump coronary bypass grafting. Eur J Cardiothorac Surg 2005;27(4):584-591

23 Carroll RC, Chavez JJ, Snider CC, Meyer DS, Muenchen RA. Correlation of perioperative platelet function and coagulation tests with bleeding after cardiopulmonary bypass surgery. J Lab Clin Med 2006;147(4):197-204

24 Preisman S, Kogan A, Itzkovsky K, Leikin G, Raanani E. Modified thromboelastography evaluation of platelet dysfunction in patients undergoing coronary artery surgery. Eur J Cardiothorac Surg 2010;37(6):1367-1374

25 Kwak YL, Kim JC, Choi YS, Yoo KJ, Song Y, Shim JK. Clopidogrel responsiveness regardless of the discontinuation date predicts increased blood loss and transfusion requirement after off-pump coronary artery bypass graft surgery. J Am Coll Cardiol 2010; 56(24):1994-2002

26 Weitzel NS, Weitzel LB, Epperson LE, Karimpour-Ford A, Tran ZV, Seres T. Platelet mapping as part of modified thromboelastography $(T E G \circledast)$ in patients undergoing cardiac surgery and cardiopulmonary bypass. Anaesthesia 2012;67(10):1158-1165

27 Mahla E, Suarez TA, Bliden KP, et al. Platelet function measurement-based strategy to reduce bleeding and waiting time in clopidogrel-treated patients undergoing coronary artery bypass graft surgery: the timing based on platelet function strategy to 
reduce clopidogrel-associated bleeding related to CABG (TARGETCABG) study. Circ Cardiovasc Interv 2012;5(2):261-269

28 Dunning J, Versteegh M, Fabbri A, et al; EACTS Audit and Guidelines Committee. Guideline on antiplatelet and anticoagulation management in cardiac surgery. Eur J Cardiothorac Surg 2008; 34(1):73-92

29 Wright RS, Anderson JL, Adams CD, et al. 2011 ACCF/AHA focused update of the Guidelines for the Management of Patients with Unstable Angina/Non-ST-Elevation Myocardial Infarction (updating the 2007 guideline): a report of the American College of Cardiology Foundation/American Heart Association Task Force on Practice Guidelines developed in collaboration with the American College of Emergency Physicians, Society for Cardiovascular Angiography and Interventions, and Society of Thoracic Surgeons. J Am Coll Cardiol 2011;57(19):1920-1959

30 Despotis GJ, Levine V, Filos KS, et al. Evaluation of a new point-ofcare test that measures PAF-mediated acceleration of coagulation in cardiac surgical patients. Anesthesiology 1996;85(6):1311-1323

31 Ereth MH, Nuttall GA, Klindworth JT, et al. Does the plateletactivated clotting test (HemoSTATUS) predict blood loss and platelet dysfunction associated with cardiopulmonary bypass? Anesth Analg 1997;85(2):259-264

32 Ereth MH, Nuttall GA, Santrach PJ, Klindworth JT, Oliver WC Jr, Schaff HV. The relation between the platelet-activated clotting test (HemoSTATUS) and blood loss after cardiopulmonary bypass. Anesthesiology 1998;88(4):962-969

33 Dietrich GV, Schueck R, Menges T, Kiesenbauer NP, Fruehauf AC, Marquardt I. Comparison of four methods for the determination of platelet function in whole blood in cardiac surgery. Thromb Res 1998;89(6):295-301

34 Wahba A, Sander S, Birnbaum DE. Are in-vitro platelet function tests useful in predicting blood loss following open heart surgery? Thorac Cardiovasc Surg 1998;46(4):228-231

35 Slaughter TF, Sreeram G, Sharma AD, El-Moalem H, East CJ, Greenberg CS. Reversible shear-mediated platelet dysfunction during cardiac surgery as assessed by the PFA-100 platelet function analyzer. Blood Coagul Fibrinolysis 2001;12(2):85-93

36 Forestier F, Coiffic A, Mouton C, Ekouevi D, Chêne G, Janvier G. Platelet function point-of-care tests in post-bypass cardiac surgery: are they relevant? Br J Anaesth 2002;89(5):715-721

37 Fattorutto M, Pradier O, Schmartz D, Ickx B, Barvais L. Does the platelet function analyser (PFA-100) predict blood loss after cardiopulmonary bypass? Br J Anaesth 2003;90(5):692-693

38 Cammerer U, Dietrich W, Rampf T, Braun SL, Richter JA. The predictive value of modified computerized thromboelastography and platelet function analysis for postoperative blood loss in routine cardiac surgery. Anesth Analg 2003;96(1):51-57 table of contents

39 Gerrah R, Brill A, Tshori S, Lubetsky A, Merin G, Varon D. Using cone and plate(let) analyzer to predict bleeding in cardiac surgery. Asian Cardiovasc Thorac Ann 2006;14(4):310-315

40 Solomon C, Hartmann J, Osthaus A, et al. Platelet concentrates transfusion in cardiac surgery in relation to preoperative point-ofcare assessment of platelet adhesion and aggregation. Platelets 2010;21(3):221-228

41 Rahe-Meyer N, Winterhalter M, Boden A, et al. Platelet concentrates transfusion in cardiac surgery and platelet function assessment by multiple electrode aggregometry. Acta Anaesthesiol Scand 2009;53(2):168-175

42 Reece MJ, Klein AA, Salviz EA, et al. Near-patient platelet function testing in patients undergoing coronary artery surgery: a pilot study. Anaesthesia 2011;66(2):97-103

43 Metz CE. Basic principles of ROC analysis. Semin Nucl Med 1978; 8(4):283-298

44 Ranucci M, Baryshnikova E, Soro G, Ballotta A, De Benedetti D, Conti D; Surgical and Clinical Outcome Research (SCORE) Group. Multiple electrode whole-blood aggregometry and bleeding in cardiac surgery patients receiving thienopyridines. Ann Thorac Surg 2011;91(1):123-129
45 Petricevic M, Biocina B, Milicic D, et al. Bleeding risk assessment using multiple electrode aggregometry in patients following coronary artery bypass surgery. J Thromb Thrombolysis 2013;35(1):31-40

46 Petricevic M, Biocina B, Milicic D, et al. Bleeding risk assessment using whole blood impedance aggregometry and rotational thromboelastometry in patients following cardiac surgery. J Thromb Thrombolysis 2013;36(4):514-526

47 Weber CF, Görlinger K, Meininger D, et al. Point-of-care testing: a prospective, randomized clinical trial of efficacy in coagulopathic cardiac surgery patients. Anesthesiology 2012;117(3):531-547

48 Schimmer C, Hamouda K, Sommer SP, Özkur M, Hain J, Leyh R. The predictive value of multiple electrode platelet aggregometry (multiplate) in adult cardiac surgery. Thorac Cardiovasc Surg 2013;61(8):733-743

49 Di Dedda U, Ranucci M, Baryshnikova E, Castelvecchio S; Surgical and Clinical Outcome Research Group. Thienopyridines resistance and recovery of platelet function after discontinuation of thienopyridines in cardiac surgery patients. Eur J Cardiothorac Surg 2014;45(1):165-170

50 Alström U, Granath F, Oldgren J, Ståhle E, Tydén H, Siegbahn A. Platelet inhibition assessed with VerifyNow, flow cytometry and PlateletMapping in patients undergoing heart surgery. Thromb Res 2009;124(5):572-577

51 Rosengart TK, Romeiser JL, White LJ, et al. Platelet activity measured by a rapid turnaround assay identifies coronary artery bypass grafting patients at increased risk for bleeding and transfusion complications after clopidogrel administration. J Thorac Cardiovasc Surg 2013;146(5):1259-1266, e1, discussion 1266

52 Petricevic M, Biocina B, Konosic S, Kopjar T, Kunac N, Gasparovic H. Assessment of platelet function by whole blood impedance aggregometry in coronary artery bypass grafting patients on acetylsalicylic acid treatment may prompt a switch to dual antiplatelet therapy. Heart Vessels 2013;28(1):57-65

53 Gurbel PA, Tantry US. Drug insight: Clopidogrel nonresponsiveness. Nat Clin Pract Cardiovasc Med 2006;3(7):387-395

54 Lordkipanidzé M, Diodati JG, Pharand C. Possibility of a rebound phenomenon following antiplatelet therapy withdrawal: a look at the clinical and pharmacological evidence. Pharmacol Ther 2009; 123(2):178-186

55 Sambu N, Warner T, Curzen N. Clopidogrel withdrawal: is there a "rebound" phenomenon? Thromb Haemost 2011;105(2):211-220

56 Görlinger K, Shore-Lesserson L, Dirkmann D, Hanke AA, RaheMeyer N, Tanaka KA. Management of hemorrhage in cardiothoracic surgery. J Cardiothorac Vasc Anesth 2013;27(4, Suppl):S20-S34

$57 \mathrm{Kim} \mathrm{JH}$, Newby LK, Clare RM, et al. Clopidogrel use and bleeding after coronary artery bypass graft surgery. Am Heart J 2008; 156(5):886-892

58 Loor G, Vivacqua A, Sabik JF III, et al. Process improvement in cardiac surgery: development and implementation of a reoperation for bleeding checklist. J Thorac Cardiovasc Surg 2013;146(5):1028-1032

59 Rahe-Meyer N, Hanke A, Schmidt DS, Hagl C, Pichlmaier M. Fibrinogen concentrate reduces intraoperative bleeding when used as first-line hemostatic therapy during major aortic replacement surgery: results from a randomized, placebo-controlled trial. J Thorac Cardiovasc Surg 2013;145(3, Suppl):S178-S185

60 Girdauskas E, Kempfert J, Kuntze T, et al. Thromboelastometrically guided transfusion protocol during aortic surgery with circulatory arrest: a prospective, randomized trial. J Thorac Cardiovasc Surg 2010;140(5):1117-1124, e2

61 Hanke AA, Herold U, Dirkmann D, Tsagakis K, Jakob H, Görlinger K. Thromboelastometry Based Early Goal-Directed Coagulation Management Reduces Blood Transfusion Requirements, Adverse Events, and Costs in Acute Type A Aortic Dissection: A Pilot Study. Transfus Med Hemother 2012;39(2):121-128

62 Lennon MJ, Gibbs NM, Weightman WM, et al. A comparison of Plateletworks and platelet aggregometry for the assessment of aspirin-related platelet dysfunction in cardiac surgical patients. J Cardiothorac Vasc Anesth 2004;18:136-140 